\title{
QUENCHED COLD ACCRETION OF A LARGE-SCALE METAL-POOR FILAMENT DUE TO VIRIAL SHOCKING IN THE HALO OF A MASSIVE $z=0.7$ GALAXY
}

\author{
Christopher W. Churchill ${ }^{1,4}$, Glenn G. Kacprzak ${ }^{2,5}$, Charles C. Steidel ${ }^{3}$, Lee R. Spitler ${ }^{2}$, \\ Jon Holtzman ${ }^{1}$, Nikole M. Nielsen ${ }^{1}$, and Sebastian Trujillo-Gomez ${ }^{1}$ \\ ${ }^{1}$ Department of Astronomy, New Mexico State University, MSC 4500, Las Cruces, NM 88003, USA \\ ${ }^{2}$ Centre for Astrophysics and Supercomputing, Swinburne University of Technology, P.O. Box 218, Hawthorn, Victoria 3122, Australia \\ ${ }^{3}$ Department of Astronomy, California Institute of Technology, MS 105-24, Pasadena, CA 91125, USA \\ Received 2012 April 17; accepted 2012 August 16; published 2012 November 6
}

\begin{abstract}
Using $H S T / C O S / S T I S$ and HIRES/Keck high-resolution spectra, we have studied a remarkable H I absorbing complex at $z=0.672$ toward the quasar Q1317+277. The H I absorption has a velocity spread of $\Delta v=1600 \mathrm{~km} \mathrm{~s}^{-1}$, comprises 21 Voigt profile components, and resides at an impact parameter of $D=58 \mathrm{kpc}$ from a bright, high-mass $\left(\log M_{\mathrm{vir}} / M_{\odot} \simeq 13.7\right)$ elliptical galaxy that is deduced to have a 6 Gyr old, solar metallicity stellar population. Ionization models suggest the majority of the structure is cold gas surrounding a shock-heated cloud that is kinematically adjacent to a multi-phase group of clouds with detected C III, C IV, and O VI absorption, suggestive of a conductive interface near the shock. The deduced metallicities are consistent with the moderate in situ enrichment relative to the levels observed in the $z \sim 3 \mathrm{Ly} \alpha$ forest. We interpret the H I complex as a metal-poor filamentary structure being shock heated as it accretes into the halo of the galaxy. The data support the scenario of an early formation period $(z>4)$ in which the galaxy was presumably fed by cold-mode gas accretion that was later quenched via virial shocking by the hot halo such that, by intermediate redshift, the cold filamentary accreting gas is continuing to be disrupted by shock heating. Thus, continued filamentary accretion is being mixed into the hot halo, indicating that the star formation of the galaxy will likely remain quenched. To date, the galaxy and the $\mathrm{H}$ I absorption complex provide some of the most compelling observational data supporting the theoretical picture in which accretion is virial shocked in the hot coronal halos of high-mass galaxies.
\end{abstract}

Key words: galaxies: evolution - galaxies: halos - quasars: absorption lines

Online-only material: color figures

\section{INTRODUCTION}

It is well accepted that galaxies are intimately linked to the gaseous cosmic web and that the evolution of galaxies is governed in large part by the dissipative response of baryonic gas due to the trade off of cooling and dynamical timescales as it accretes into dark matter halos (e.g., Binney 1977; Rees \& Ostriker 1977; Silk 1977; White \& Rees 1978). In general, distinct modes of accretion are believed to operate and the mode is dependent primarily upon the dark matter halo mass (e.g., Birnboim \& Dekel 2003; Dekel \& Birnboim 2006), with some dependence on environment (e.g., Kereš et al. 2005) and on feedback (e.g., van de Voort et al. 2011).

"Hot-mode" accretion is the mechanism in which inflowing gas is shock heated as it is compressed by the hot hydrostatic gas halo. This mode dominates around high-mass galaxies, where the dynamical time is shorter than the cooling time. For the most part, the gas accretes into the halo, but not necessarily onto the galaxy itself. "Cold-mode" accretion is the mechanism primarily around low-mass galaxies, where the cooling time is shorter than the dynamical time so that hot hydrostatic halos do not form and cold gas accretes directly onto the galaxy. At lower redshifts, as densities decrease, the cooling time is generally longer than the dynamical time and the rate of infalling material decreases so that cold-mode accretion is a minor channel of accretion onto galaxies (e.g., Kereš et al. 2005; Dekel \& Birnboim 2006),

\footnotetext{
4 Visiting Professor, Swinburne University of Technology, Victoria 3122, Australia.

5 Australian Research Council Super Science Fellow.
}

though it remains an important channel for the growth of galaxies at all redshifts (e.g., van de Voort et al. 2011).

A possible third mode is the case in which cold dense filaments can penetrate directly into a hot halo of a massive galaxy. If the conditions are favorable for short cooling times in the filament, then shock heating can be avoided and the filamentary gas can directly accrete onto the galaxy (e.g., Dekel \& Birnboim 2006; Ocvirk et al. 2008; Dekel et al. 2009; van de Voort \& Schaye 2012). However, though cold streams can penetrate the hot atmospheres of massive halos at $z \geqslant 2$, this process significantly diminishes at lower redshift (e.g., Ocvirk et al. 2008; Kereš et al. 2009; Faucher-Giguère et al. 2011), though there is plausible evidence of this process occurring in some $z<1$ galaxies (Kacprzak et al. 2011,2012; Ribaudo et al. 2011; Thom et al. 2011). See Dekel et al. (2009) for additional insights.

One observable signature of a filament may be a "complex" of $\mathrm{H} \mathrm{I}$ absorption with a large velocity spread $\left(\Delta v>1000 \mathrm{~km} \mathrm{~s}^{-1}\right)$. Alternatively, such $\mathrm{HI}$ absorbing complexes may trace the warm-hot ionized medium (WHIM), or the intracluster and/or intragroup medium. If an observed $\mathrm{H}$ I absorption complex arises from a filament, it is plausible that the filament may be accreting from the intergalactic medium (IGM) into a galaxy halo or even directly onto a galaxy. Thus, Hi absorption complexes provide unique astrophysical laboratories for placing constraints on our understanding of the IGM, and the processes giving rise to extended galaxy halos in the context of dark matter overdensities.

At high redshifts $(z>2)$, kinematically extended $\mathrm{H}$ I absorbing complexes were studied by Cowie et al. (1996) with 
Keck/HIRES spectra. They deduced that these structures were filamentary in nature. At lower redshift $(z<0.22)$, Shull et al. (1998, 2003), Tripp et al. (2001), and Aracil et al. (2006a) studied three different $\mathrm{HI}_{\mathrm{I}}$ complexes with high-resolution ultraviolet spectra in which metal lines were detected. For these three complexes, several galaxies were found in the vicinity, suggesting moderate size groups. Each of these studies favored a different scenario of explanation for the physical picture of the H I complex, including O VI arising in "nearside/backside" shocked infall into the potential well of the galaxy group (Shull et al. 2003), intragroup gas or an unvirialized filamentary structure through the group (Tripp et al. 2001), and tidally stripped material from one of the nearby galaxies (Aracil et al. 2006a).

The spectrum of the quasar Q1317+277 (TON 153, CSO $\left.0873, \mathrm{~J} 131956+272808, V=16.0, z_{\mathrm{em}}=1.017\right)$ exhibits a dramatic $\Delta v>1000 \mathrm{~km} \mathrm{~s}^{-1} \mathrm{HI}$ complex observed in Ly $\alpha$ absorption at $z=0.672$. Several optical and ultraviolet spectra of the quasar are available and have been the focus of this $\mathrm{H}$ I complex and/or the Lyman limit metal-line system at $z=0.660$ (Steidel \& Sargent 1992; Bahcall et al. 1993, 1996; Churchill et al. 2000, 2007, 2012; Ding et al. 2005; Kacprzak et al. 2011, 2012). Besides being at intermediate redshift, what is unique to this $\mathrm{H}$ I complex is that it lies at $58 \mathrm{kpc}$ projected from a single bright elliptical galaxy at $z_{\mathrm{gal}}=0.6719$ (Churchill et al. 2007).

An analysis of the $z=0.672 \mathrm{HI}$ complex was presented in Churchill et al. (2007, hereafter Paper I) based upon HST G160L/G190H FOS spectra (PID 2424; PI: J. N. Bahcall), an HST E230M/STIS spectrum (PID 8672; PI: Churchill), and a Keck/HIRES spectrum of the quasar (see Churchill 1997). In the Faint Object Spectrograph (FOS) spectrum, the H I complex was found to comprise five components of optically thin $\mathrm{H}$ I absorbing gas, which span a velocity range of $\Delta v=1400 \mathrm{~km} \mathrm{~s}^{-1}$. No metal lines were clearly detected in the FOS, Space Telescope Imaging Spectrograph (STIS), and High Resolution Echelle Spectrometer (HIRES) spectra.

Galaxies at the absorber redshifts were first reported by Steidel et al. (1994) as part of their Mg II absorption-selected galaxy survey. The quantified morphological and spectral properties of these galaxies were presented in Paper I based upon an HST/WFPC2 F702W image (PID 5984; PI: Steidel) of the quasar field and Keck/LRIS spectra of the galaxies. Updated analysis of the galaxies is included in Kacprzak et al. (2011, 2012) and Churchill et al. (2012). In summary, the galaxy at the redshift of the H I complex has $z_{\mathrm{gal}}=0.6719$ and an impact parameter of $D=58 \mathrm{kpc}$. It is classified as a late-type E/S0 galaxy. The galaxy at the redshift of the $z=0.660$ absorber, which is a rich metal-line Lyman limit system, has $z_{\text {gal }}=0.6610$, an impact parameter of $D=104$, and is an inclined Sab galaxy.

In Paper I, we deduced that the gas in the Hi complex has column densities in the range $14.5 \leqslant \log N(\mathrm{HI}) \leqslant 15.5$, temperatures in the range $5.0 \leqslant \log T \leqslant 5.5$, and upper limits on metallicity in the range $-1.0 \leqslant \log Z / Z_{\odot} \leqslant-3.0$. We further deduced that the complex is consistent with a combination of photo- and collisionally ionized gas. Based upon expectations of simulations (e.g., Davé et al. 1999), we favored a shock-heated structure with chemical enrichment consistent with the high-redshift IGM. Our interpretation was that the $\mathrm{H}$ I complex is a shock-heated filamentary structure that originated as a photoionized diffuse phase of gas at high redshift and is accreting in the vicinity of the $z_{\mathrm{gal}}=0.6719$ elliptical galaxy.

However, the FOS data did not provide the information required to examine the detailed structure of the kinematic and ionization conditions. Thus, we were unable to constrain the kinematic relationships between the putative shock-heated gas, the photo- and/or collisionally ionized gas, and the $z_{\mathrm{gal}}=$ 0.6719 galaxy. Such information is critical for examining the physics of gas accretion onto galaxies and for comparing with other observations and with cosmological simulations. For example, Cowie et al. (1996) claim that clustered Ly $\alpha$ lines at $z=3$ are observed to have higher ionization conditions at the velocity extremes and suggest this layered structure is a signature of collapsing structures. While this may be a signature for intergalactic filaments, it is not clear that accretion of a filament into a galaxy potential will yield the same velocityionization structure. In theoretical treatments (e.g., Birnboim \& Dekel 2003; Dekel \& Birnboim 2006; Birnboim et al. 2007) and cosmological simulations (e.g., Kereš et al. 2005, 2009; van de Voort et al. 2011; van de Voort \& Schaye 2012) accretion onto massive galaxies is expected to shock heat upon entry into a hot coronal halo, and the resulting kinematic-ionization structure may reflect this very different process.

In order to better study the Q1317+277 $\mathrm{HI}$ complex at $z=0.672$, we have obtained a high-resolution $R=18,000$ $H S T / C O S$ spectrum of the quasar, with focus on the H I Lyman series lines and $\mathrm{O} v \mathrm{VI} \lambda \lambda 1031,1037$ absorption. We further improved our knowledge of the star formation history, age, and mass of the $z_{\mathrm{gal}}=0.6719$ galaxy by obtaining multiband imaging of the quasar field. Our motivations include (1) a thorough examination of the kinematic, ionization, and chemical structure of the H I complex, (2) a direct comparison with the galaxy properties to help place the galaxy-absorber system in the context of galaxy evolution scenarios predicted by theories and cosmological simulations for an improved interpretation, and (3) examination whether there is a connection between the $z=0.672 \mathrm{H}$ i complex and the galaxy-absorber pair at $z=0.661$, perhaps in the form of a "bridge" of weak Ly $\alpha$ absorption between the galaxies.

The paper is structured as follows: reduction and analysis of the imaging and spectroscopic data are presented in Section 2. Our analysis of the galaxy images is presented in Section 3 and our analysis of the absorption line data is presented in Section 4. In Sections 5 and 6, we present our ionization modeling and resulting constraints on the physical conditions of the absorbing gas. We discuss and interpret the data and our findings in Section 7. We provide brief a conclusion in Section 8. Throughout this work, we assume the cosmological parameters $\Omega_{\Lambda}=0.7, \Omega_{m}=0.3, \Omega_{k}=0$, and $h=$ $H_{0} /\left(100 \mathrm{~km} \mathrm{~s}^{-1} \mathrm{Mpc}^{-1}\right)=0.7$ (based on Jarosik et al. 2011).

\section{OBSERVATIONS, REDUCTIONS, AND CALIBRATIONS}

We have acquired new data on the $z=0.660$ and $z=0.672$ absorption and the two galaxies G1 and G2. First, we obtained a cycle-17 $R \simeq 18,000 \operatorname{COS}$ (Cosmic Origins Spectrograph) spectrum (PID 11667; PI: Churchill) of the quasar covering the transitions examined in the FOS spectra. Our goals include measuring higher detection sensitivities and detailed kinematics of the absorption lines. Second, we obtained $g^{\prime}, r^{\prime}, i^{\prime}$, and $K_{s}$ ground-based images of the quasar field. The multi-band images provide colors from which galaxy stellar populations, metallicities, and masses can be estimated.

\subsection{Ground-based Images}

As part of a larger campaign for imaging $\mathrm{Mg}$ II absorption galaxies, we obtained $g^{\prime}-, r^{\prime}-$, and $i^{\prime}$-band images of the quasar 
field using the Seaver Prototype Imaging camera (SPIcam) on the Apache Point Observatory's (APO) $3.5 \mathrm{~m}$ telescope. The detector is a $2048 \times 2048 \mathrm{CCD}$ with $24 \mu \mathrm{m}$ pixels, giving an unbinned plate scale of $0^{\prime \prime} 14$ pixel $^{-1}$ and a field of view of $4^{\prime} .78 \times 44^{\prime} .78$. We binned the CCD $2 \times 2$ during readout for a plate scale of $0^{\prime \prime} .28$ pixel $^{-1}$. Multiple $g^{\prime}-, r^{\prime}$-, and $i^{\prime}$-band images were obtained on the nights of 2006 March 24-25, for which the seeing varied between $1^{\prime \prime} .2-1$.'.5, and additional $g^{\prime}$-band images were obtained the night of 2007 March 15 for which the seeing was $0{ }^{\prime \prime} 7$. The total summed exposure times are 5190, 4630, and $4350 \mathrm{~s}$ for the $g^{\prime}, r^{\prime}$, and $i^{\prime}$ filters, respectively.

Each frame was reduced using standard IDL and IRAF scripts and tasks. Flat fielding incorporated a combination of dome and sky flats. Cosmic rays were removed in each individual frame. The astrometry was calibrated by position matching of USNO A2.0 stars in the field. Final images were obtained by co-adding the individual calibrated frames.

The photometric zero points were obtained using stars in Sloan Digital Sky Survey (SDSS) images. Color terms are required because the filter plus detector throughput of the APO facility is not identical to that of the SDSS facility. These color terms, which are of order 0.1, were determined from SPIcam and SDSS images of roughly 30 quasar fields from our more extensive database.

The near-infrared band images were obtained on 1994 February 24 using the Kitt Peak Mayall $4 \mathrm{~m}$ telescope through the $K_{s}$ filter $(1.99-2.32 \mu \mathrm{m})$ with the IRIM NICMOS III $256 \times 256$ array camera. These images were obtained as part of the campaign culminating in the work of Steidel et al. (1994). The field of view is $154^{\prime \prime} \times 154^{\prime \prime}$ with plate scale of $0^{\prime \prime} 6$ pixel $^{-1}$. The NICMOS images were reduced using the contributed IRAF package DIMSUM. ${ }^{7}$ Updated photometric zero points were determined using stars from the 2MASS point-source catalog (Strutskie et al. 2006).

All photometric measurements were conducted using SExtractor (Bertin \& Arnouts 1996); we adopted the AUTOMAG results. The dust maps of Schlegel et al. (1998) were used to correct for Galactic dust extinction.

In Figures 1(a)-(d), we present $43^{\prime \prime} \times 60^{\prime \prime}$ sections of the ground-based images centered on the quasar Q1317+277. A $12^{\prime \prime} \times 22^{\prime \prime}$ section of the HST/WFPC2 F702W image is presented as Figure 1(e). Galaxies G1 and G2 are labeled in all images. In the Hubble Space Telescope (HST) image, note the object to the north by northeast within $2^{\prime \prime} .1$ of the quasar. We have no estimate of the redshift of this object, which we label G3. Reduction and analysis of the F702W image was described in Paper I, Kacprzak et al. (2011), and Churchill et al. (2012). Expanded views of the two galaxies are presented in Figures 1(f) and (g). The Keck/LRIS spectra of galaxies G1 and G2 (originally described in Paper I) are presented in Figures 1(h) and (i). The G1 galaxy redshift was determined using Gaussian centroiding to the Ca II absorption features and the redshift of $\mathrm{G} 2$ was determined using Gaussian centroiding to the [O II] $\lambda 3727$ emission line.

\subsection{HST/COS Spectrum}

We obtained a cycle-17 $R \simeq 18,000$ COS spectrum (PID 11667; PI: Churchill) of the quasar covering the transitions first

\footnotetext{
6 IRAF is written and supported by the IRAF programming group at the National Optical Astronomy Observatories (NOAO) in Tucson, Arizona. NOAO is operated by the Association of Universities for Research in Astronomy (AURA), Inc., under cooperative agreement with the National Science Foundation.

7 http://iraf.noao.edu/iraf/ftp/contrib/dimsumV3/. DIMSUM was contributed by P. Eisenhardt, M. Dickinson, S. A. Stanford, \& F. Valdez.
}

examined in the FOS spectra. Two NUV/G185M spectra were obtained on 2010 May 26 and optimally co-added. The first was centered at $1921 \AA$ for a 5420 s exposure, and the second was centered at $1941 \AA$ for a 4970 s exposure. The overlap region was 2223-2037 $\AA$ on Stripe C, which provided a total of $10,390 \mathrm{~s}$ of integration on the $\mathrm{H}$ I absorption complex. The FUV/G160M spectrum was obtained on 2010 June 26 centered at $1600 \AA$ for a $12,580 \mathrm{~s}$ exposure.

The spectra were reduced following the procedures outlined by Shaw et al. (2009). We continuum fitted the lower order shape of the spectrum using the IRAF sfit task and refined the higher order continuum features using our own code Fitter (Churchill et al. 2000).

For the $z=0.660$ and $z=0.672$ absorbers, the $\operatorname{Ly} \beta$ and $\operatorname{Ly} \gamma$ absorption lines were captured in the NUV on Segment A, the Ly $\delta$ was not captured (fell between segments), and the higher order Lyman series lines were captured on Segment B.

\section{IMAGE ANALYSIS: GALAXY PROPERTIES}

A re-analysis ${ }^{8}$ of the $H S T /$ WFPC2 F702W image was undertaken, presented, and fully described by Churchill et al. (2012). We adopt the measured quantities from that work.

The G1 quasar-galaxy impact parameter is $D=58 \mathrm{kpc}$. The galaxy photometric properties are $M_{B}=-21.6, M_{K}=-23.0$, and $B-K=1.4(\mathrm{AB})$. The $B$-band luminosity is $L_{B} / L_{B}^{*}=$ 1.28 , where we use $M_{B}^{*}$ from the fit with redshift reported by Faber et al. (2007). From an analysis using GIM2D (Simard et al. 2002), we measure a half-light radius of $r_{h}=4.2 \mathrm{kpc}$, disk scale length of $r_{d}=0.9 \mathrm{kpc}$, and bulge-to-total ratio of $B / T=0.99$. The galaxy classifies as an $\mathrm{E} / \mathrm{S} 0$ based upon its C-A morphology (Abraham et al. 1996). The galaxy inclination is $i=15.9$, and the angle between the quasar sight line and the major axis of the projected ellipse of the galaxy is $\theta=22^{\circ} .1$. For the properties of galaxy G2, see Churchill et al. (2012) and our companion paper (Kacprzak et al. 2012).

Photometric analysis of the ground-based images yielded dust-, color-, and seeing-corrected $\mathrm{AB}$ apparent magnitudes for galaxy $\mathrm{G} 1$ of $m_{g^{\prime}}=23.4 \pm 0.03, m_{r^{\prime}}=22.2 \pm 0.03$, $m_{i^{\prime}}=21.01 \pm 0.03$, and $m_{K_{s}}=19.4 \pm 0.1$. A color composite image of the ground-based images can be viewed in our companion paper (Kacprzak et al. 2012), from which it can be ascertained that galaxy G1 is clearly redder than galaxy G2 and that the other galaxies in the field (see Figures 1(a)-(d)) are likely at substantially different redshifts.

For galaxy G3, we measured an F702W apparent magnitude of $m=23.4 \pm 0.2$. This value is based upon a dithered coadded image constructed by A. Shapley, in which she removed the quasar via point-spread function (PSF) subtraction. The quoted uncertainty is statistical based upon the sky background; due to the PSF subtraction, the error could be substantially underestimated. Assuming galaxy G3 is at the redshift of the $\mathrm{H}_{\mathrm{I}}$ absorption complex, i.e., $z \simeq 0.672$, and adopting the quoted apparent magnitude, we compute $M_{B}=-19.3$ $\left(L_{B} / L_{B}^{*}=0.16\right)$ and $M_{r}=-20.7$ (SDSS $r$ band). At this redshift, the impact parameter to $\mathrm{G} 3$ is $D=14.6 \mathrm{kpc}$.

In Figure 2, we plot the $g^{\prime}-r^{\prime}$ colors versus $i^{\prime}-K_{s}$ colors for G1 and G2. Following Bell et al. (2003), Fontana et al. (2004), and Swindle et al. (2011), we used stellar population models to determine stellar masses, $M_{*}$, of galaxies G1 and G2 from the observed colors. We employed the stellar population

\footnotetext{
8 We note that an incorrect $k$-correction resulted in an overestimate of $M_{B}$ and $L_{B} / L_{B}^{*}$ in Paper I. We also converted all magnitudes to the AB system.
} 

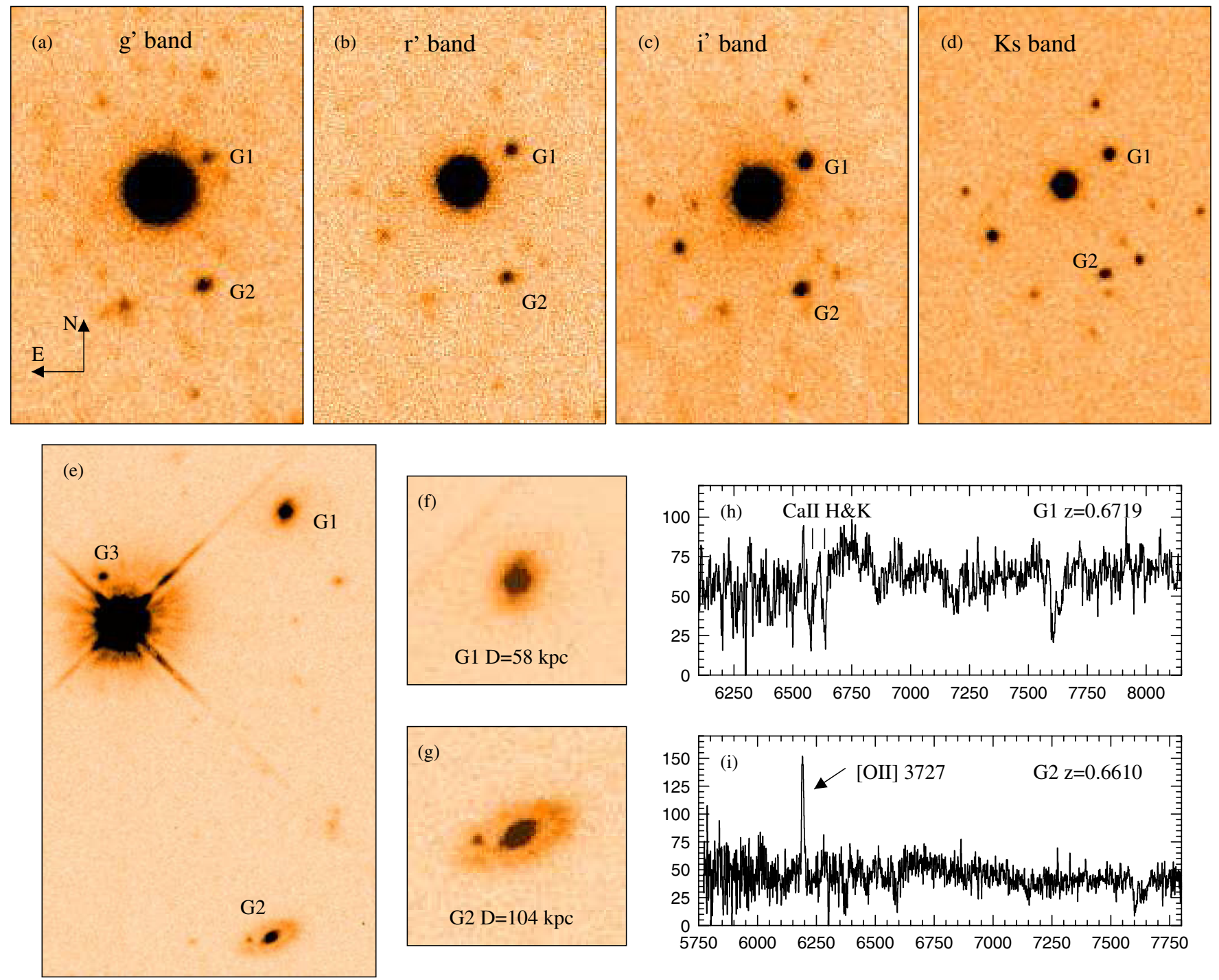

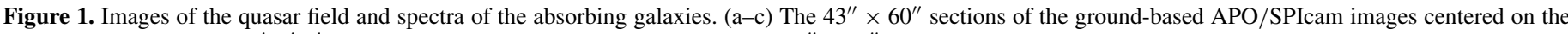

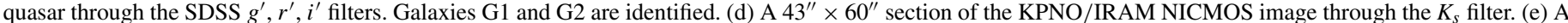

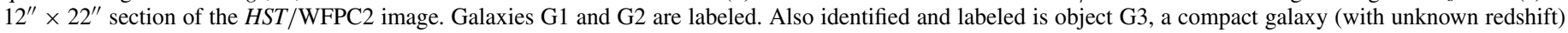

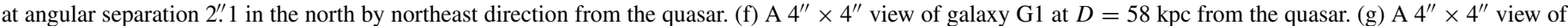

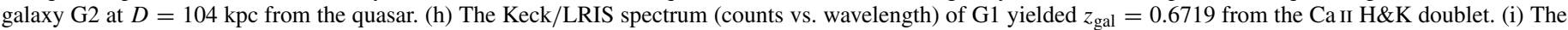
Keck/LRIS spectrum (counts vs. wavelength) of G2 yielded $z_{\text {gal }}=0.6610$ from the $[\mathrm{O}$ II $] \lambda 3727$ emission line.

(A color version of this figure is available in the online journal.)

models of Bruzual \& Charlot (2003) assuming a Chabrier (2003) initial mass function and an exponential star formation history with an $e$-folding time of $1 \mathrm{Gyr}^{9}{ }^{9}$ Also shown in Figure 2 are the loci of observed colors as a function of redshift for the Bruzual \& Charlot (2003) stellar population models for the metallicities $[Z / \mathrm{H}]=-0.4,0.0$, and +0.4 . We find galaxy G1 is consistent with a $\simeq 5.8 \mathrm{Gyr}$ old, solar metallicity stellar population with a formation epoch of $z=4$. The stellar population models also yield the galaxy $K$-band mass-to-light ratio, $(M / L)_{K}$. From this ratio and the $K$-band magnitude, we estimate $\log M_{*} / M_{\odot}=\log (M / L)_{K}-0.4\left(M_{K}-M_{K, \odot}\right)=11.5$ for galaxy G1, where $M_{K, \odot}=-3.28$ is the solar value.

Using the technique of halo abundance matching, the galaxy virial mass, $M_{\text {vir }}$, can be estimated from the stellar mass (e.g., Conroy \& Wechsler 2009; Behroozi et al. 2010; Moster et al. 2010; Stewart 2011). Abundance matching assumes a

\footnotetext{
9 The stellar population models were generated using the web service
} EZGAL (www.baryons.org/ezgal). monotonic functional relation between $M_{\mathrm{vir}}$ and $M_{*}$ by assigning the number of halos with $M_{\text {halo }}>M_{\text {vir }}$ equal to the number of galaxies with $M_{\text {gal }}>M_{*}$. As such, it matches the halo mass and stellar mass functions globally with a roughly 0.25 dex uncertainty in $M_{\text {vir }}$ at fixed $M_{*}$, primarily due to the systematics in estimates of $M_{*}$ (Behroozi et al. 2010). We employed the parameterized functions presented by (Stewart 2011).

For galaxy G1, we obtained $M_{\mathrm{vir}}=10^{13.7}-10^{14.4} M_{\odot}$, where the lower value is given by the Conroy \& Wechsler (2009) and Moster et al. (2010) fits and the higher value is from the Behroozi et al. (2010) fit. ${ }^{10}$ We adopted the Conroy \& Wechsler (2009) and Moster et al. (2010) values $M_{\text {vir }}=10^{13.7} M_{\odot}$.

\footnotetext{
${ }^{10}$ Abundance matching is the most accurate for $M_{\mathrm{vir}}=10^{11}-10^{13} M_{\odot}$. For less massive halos, the stellar mass function is not tightly constrained. For higher mass halos, particularly massive ellipticals, there is substantial scatter between the published abundance matching predictions/relations. Thus, for a single case, abundance matching is not highly robust for mapping $M_{\mathrm{vir}}$ from $M_{*}$ when $M_{\text {vir }}>10^{13} M_{\odot}$ (K. R. Stewart 2012, private communication).
} 


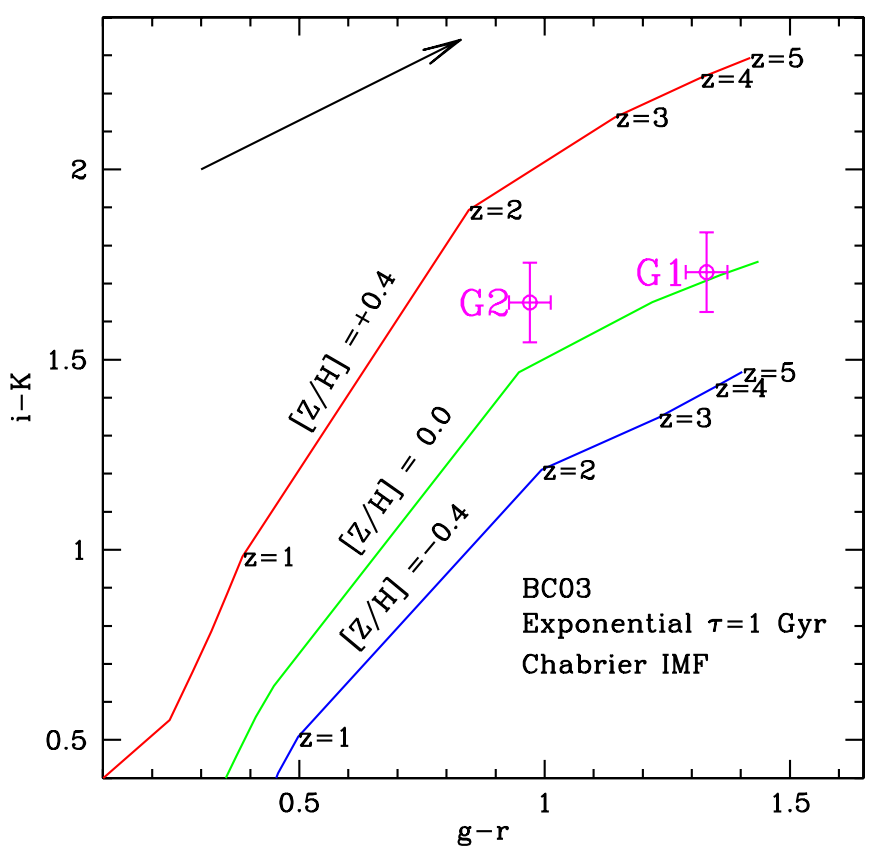

Figure 2. $g^{\prime}-r^{\prime}$ vs. $i^{\prime}-K_{s}$ color-color diagram showing the measured dustcorrected colors of galaxies G1 and G2. The curves are Bruzual \& Charlot (2003) stellar population models for metallicities $[Z / H]=-0.4,0.0$, and +0.4 assuming an exponential star formation rate with an $e$-folding time of $1 \mathrm{Gyr}$ and a Chabrier (2003) initial mass function. The arrow provides the reddening vector for $E(B-V)=0.1$ in the rest frame of the galaxies.

(A color version of this figure is available in the online journal.)

Under the assumption that galaxy G3 resides at $z=0.672$, we use the $\Lambda$ CDM Bolshoi Simulation Database of Trujillo-Gomez et al. (2011) to estimate a virial mass of $M_{\mathrm{vir}}=10^{11.9} M_{\odot}$ using abundance matching. This mass is the average of 18,500 galaxies in the absolute magnitude bin $-21.7 \leqslant M_{r} \leqslant-19.7$ with average $M_{r}=-20.7$. The abundance matching in this database is constrained by the observed luminosity-circularvelocity relation, the baryonic Tully-Fisher relation, and the circular velocity function, allowing all types of galaxies to be included. Using the parameterized abundance matching function for $M_{*}\left(M_{\mathrm{vir}}, z\right)$ of Moster et al. (2010), we estimate that G3 has a stellar mass of $M_{*} \simeq 10^{10.2} M_{\odot}$.

The average gas mass, $M_{\text {gas }}$, of a galaxy with stellar mass $M_{*}$ can be estimated using the parameterized relation of Stewart (2011) based upon the baryonic Tully-Fisher relation study of McGaugh (2005), the stellar, gas, and dynamical mass relations of Erb et al. (2006), and galaxy gas fraction stellar mass study of Stewart et al. (2009). We obtain $M_{\mathrm{gas}}=10^{10.2+0.2} M_{\odot}$ for galaxy G1 ${ }^{11}$ and estimate $M_{\text {gas }}=10^{9.9 \pm 0.3} M_{\odot}$ for G3. Thus, we deduce averaged baryonic gas fractions, $f_{g}=M_{\mathrm{gas}} /\left(M_{*}+M_{\mathrm{gas}}\right)$, of $5 \%$ for $\mathrm{G} 1$ and $34 \%$ for $\mathrm{G} 3$.

The virial radii, virial temperatures, and circular velocities for galaxies $\mathrm{G} 1$ and $\mathrm{G} 3$ were computed using the relations of Bryan $\&$ Norman (1998) for $\Omega_{\mathrm{R}}=0$. For galaxy G1, we obtained $R_{\text {vir }}=750 \mathrm{kpc}, T_{\mathrm{vir}}=1 \times 10^{7} \mathrm{~K}$, and $v_{\text {circ }}=550 \mathrm{~km} \mathrm{~s}^{-1}$ and for galaxy G3, we obtained $R_{\text {vir }}=180 \mathrm{kpc}, T_{\mathrm{vir}}=7 \times 10^{5} \mathrm{~K}$, and $v_{\text {circ }}=150 \mathrm{~km} \mathrm{~s}^{-1}$. In Table 1, we summarize the deduced

\footnotetext{
11 The estimates of the gas mass is based upon the $M_{\mathrm{gas}}-M_{*}$ correlation for disk galaxies, and is therefore not directly applicable to elliptical galaxies. However, as shown in Figure 2 of Stewart et al. (2009), for $M_{*}>10^{11} M_{\odot}$, the gas-poor disk galaxy gas fractions reasonably match those of the red galaxy sample of Kannappan (2004). Thus, we can crudely apply the relation to $M_{*}>10^{11} M_{\odot}$ elliptical galaxies (K. R. Stewart 2012, private communication).
}

Table 1

Galaxy Properties

\begin{tabular}{lccc}
\hline \hline Property & $\mathrm{G} 1$ & $\mathrm{G}^{\mathrm{a}}$ & $\mathrm{G}^{\mathrm{b}}$ \\
\hline$M_{*} / M_{\odot}$ & $3 \times 10^{11}$ & $1 \times 10^{11}$ & $1 \times 10^{10}$ \\
$M_{\text {vir }} / M_{\odot}$ & $5 \times 10^{13}$ & $8 \times 10^{12}$ & $8 \times 10^{11}$ \\
$T_{\text {vir }}(\mathrm{K})$ & $1 \times 10^{7}$ & $3 \times 10^{6}$ & $7 \times 10^{5}$ \\
$v_{\text {circ }}\left(\mathrm{km} \mathrm{s}^{-1}\right)$ & 550 & 280 & 150 \\
$R_{\text {vir }}(\mathrm{kpc})$ & 750 & 380 & 180 \\
$M_{\text {gas }} / M_{\odot}$ & $2 \times 10^{10}$ & $1 \times 10^{10}$ & $7 \times 10^{9}$ \\
$M_{\text {bary }} / M_{\odot}$ & $3.2 \times 10^{11}$ & $1.1 \times 10^{11}$ & $1.7 \times 10^{10}$ \\
$f_{g}$ & 0.05 & 0.09 & 0.34 \\
\hline
\end{tabular}

Notes.

a Taken from Kacprzak et al. (2012).

${ }^{\mathrm{b}}$ Assuming $z=0.672$.

galaxy properties, which are representative averages for galaxies with their observed photometric properties.

\section{SPECTRAL ANALYSIS: ABSORPTION PROPERTIES}

In the FOS spectrum, a very broad H I absorption complex was observed (first reported by Bahcall et al. 1993). In Paper I, we fitted the Ly $\alpha$ with five Gaussian components at redshifts $z=$ $0.66914,0.67157,0.67355,0.67559$, and 0.67707 , respectively. The total rest-frame equivalent width was determined to be $W_{r}(\mathrm{Ly} \alpha)=2.87 \AA$ over a rest-frame velocity spread of $1420 \mathrm{~km} \mathrm{~s}^{-1}$. From the STIS spectrum, the $3 \sigma$ C IV $\lambda 1548$ equivalent width limit was estimated to be $W_{r}(1548) \leqslant 0.03 \AA$ at $z=0.6719$. In the FOS spectrum, the $3 \sigma$ O vi $\lambda 1031$ equivalent width limit was estimated to be $W_{r}(1031) \leqslant 0.21 \AA$. For Mg II $\lambda 2796$ in the HIRES spectrum, we obtained $W_{r}(2796) \leqslant 7 \mathrm{~m} \AA$ to $3 \sigma$. These values assumed unresolved absorption.

For this work, we objectively locate absorption lines or place limits on their equivalent widths employing the optimized methods of Schneider et al. (1993) and Churchill et al. (1999b) as modified using the methods for unresolved lines and pattern noise developed by Lawton et al. (2008). We adopt a $5 \sigma$ detection threshold and quote $3 \sigma$ limits.

\subsection{Neutral Hydrogen Lines}

In Figure 3, we present the $\operatorname{Ly} \alpha, \operatorname{Ly} \beta, \operatorname{Ly} \gamma, \operatorname{Ly} \epsilon$, and $\operatorname{Ly} \zeta$ absorption observed in the COS spectrum as a function of restframe velocity relative to the $z_{\text {gal }}=0.6719$ galaxy. No H I absorption was detected for Lyman series lines higher than Ly $\zeta$. There is significant blending in the $\operatorname{Ly} \beta, \operatorname{Ly} \gamma, \operatorname{Ly} \epsilon$, and $\operatorname{Ly} \zeta$ lines, which are identified in Figure 3, when possible.

The curves through the data are $\chi^{2}$ minimized Voigt profile (VP) fits obtained using our code MINFIT (Churchill 1997; Churchill \& Vogt 2001; Churchill et al. 2003). The ticks above the normalized continuum provide the VP component velocities. During the fitting, the COS instrumental line spread function (ISF) was convolved with the VP model. The COS ISF appropriate for the spectrograph settings and the observed wavelength of each transition was determined via interpolation of the online tabulated data (cf. Dixon et al. 2011; Kriss 2011).

For the VP fitting, pixels compromised by blending were masked out of the least-squares fit vector. We present the results of our VP modeling in Table 2. We fitted a total of 21 components, or "clouds" (MINFIT returns the minimum number of components based upon their statistical significance through a series of $F$-tests and confidence level checks). Assuming thermal 

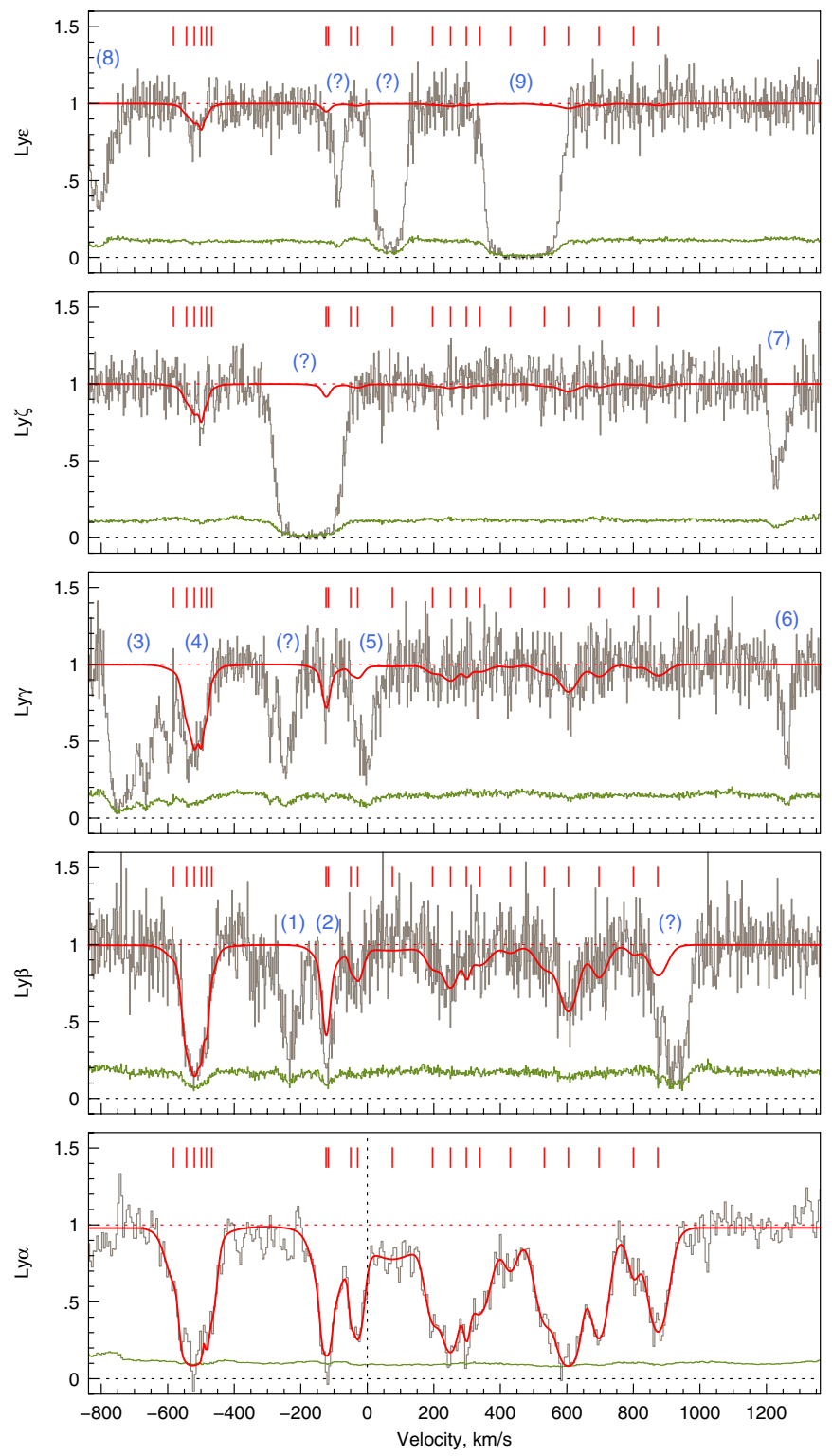

Figure 3. Voigt profile decomposition of the $\mathrm{H}$ I complex, including $\operatorname{Ly} \beta$, Ly $\gamma$, $\operatorname{Ly} \zeta$, and Ly $\epsilon$ (Ly $\delta$ was not captured). The vertical dashed line is the redshift of galaxy G1. The component parameters' centroids are marked by ticks above the continuum and the fitted component parameters are listed in Tables 2 and 3. Several blends are present and identified as $(1,2) \mathrm{O}$ VI $\lambda 1031$ associated with the $z=0.6610$ metal system, (3,4) C III $\lambda 977$ at $z=0.6610,(5) \mathrm{Ly} \delta$ at $z=0.7738$, (6) $C_{\text {III }} \lambda 977$ from clouds 7 and 8 of the H I complex, (7) Ly $\beta z=0.5349$, (8) Galactic C IV $\lambda 1550$, and (9) Ly $\zeta$ at $z=0.6610$. Those marked with "(?)" may well be Ly $\alpha$ lines in that there is no corroborating data to suggest they are metal lines nor higher order Lyman series lines.

(A color version of this figure is available in the online journal.)

broadening, we converted the Doppler $b$ parameter into the "cloud" temperature.

The strong Ly $\alpha$ absorption centered at $v \simeq-530 \mathrm{~km} \mathrm{~s}^{-1}$ $(z \simeq 0.6690)$ is blended with $\operatorname{Ly} \beta$ at $z=0.9776$ associated with the Ly $\alpha$ absorption identified by Bahcall et al. (1996) at $z=0.9778$ in the FOS spectrum, which we confirmed in the STIS spectrum. Prior to VP fitting the H I complex, we deblended the $v \simeq-530 \mathrm{~km} \mathrm{~s}^{-1}$ Ly $\alpha$ feature using VP modeling and employing both the COS and STIS ISFs. In Figure 4(a), we present the VP fits at $z=0.97763$. The flux decrement of the $\operatorname{Ly} \beta$ VP model was then subtracted from that of the
Table 2

Voigt Profile Decomposition of the H I Complex

\begin{tabular}{lccccc}
\hline \hline Cloud No. & $z_{\mathrm{cl}}$ & $\begin{array}{c}v_{\mathrm{cl}^{\mathrm{a}}} \\
\left(\mathrm{km} \mathrm{s}^{-1}\right)\end{array}$ & $\log N(\mathrm{HI})$ & $\begin{array}{c}b(\mathrm{HI}) \\
\left(\mathrm{km} \mathrm{s}^{-1}\right)\end{array}$ & $\begin{array}{c}T \\
\left(10^{3} \mathrm{~K}\right)\end{array}$ \\
\hline $1^{\mathrm{b}}$ & 0.668645 & -582.1 & $13.24 \pm 0.59$ & $31.9 \pm 31.3$ & $62.0 \pm 60.9$ \\
$2^{\mathrm{b}}$ & 0.668861 & -543.5 & $14.26 \pm 0.54$ & $14.7 \pm 9.9$ & $13.2 \pm 8.9$ \\
$3^{\mathrm{b}}$ & 0.669000 & -518.6 & $14.53 \pm 0.33$ & $12.8 \pm 10.7$ & $10.0 \pm 8.4$ \\
$4^{\mathrm{b}}$ & 0.669112 & -498.5 & $14.51 \pm 0.14$ & $3.2 \pm 2.1$ & $0.6 \pm 0.4$ \\
$5^{\mathrm{b}}$ & 0.669199 & -482.9 & $14.07 \pm 0.20$ & $4.0 \pm 1.8$ & $1.0 \pm 0.4$ \\
$6^{\mathrm{b}}$ & 0.669290 & -520.6 & $13.38 \pm 0.38$ & $15.5 \pm 11.2$ & $14.7 \pm 10.5$ \\
$7^{3}$ & 0.671203 & -123.8 & $14.13 \pm 0.10$ & $11.5 \pm 2.9$ & $8.1 \pm 2.0$ \\
$8^{2}$ & 0.671244 & -116.6 & $13.69 \pm 0.14$ & $43.3 \pm 14.4$ & $114.5 \pm 38.1$ \\
9 & 0.671617 & -49.6 & $12.93 \pm 0.94$ & $5.8 \pm 15.5$ & $2.1 \pm 5.5$ \\
10 & 0.671739 & -27.7 & $13.70 \pm 0.20$ & $18.3 \pm 7.7$ & $20.5 \pm 8.6$ \\
11 & 0.672319 & +76.1 & $13.43 \pm 0.19$ & $88.6 \pm 49.2$ & $479.3 \pm 266.1$ \\
12 & 0.672994 & +197.0 & $13.59 \pm 0.28$ & $26.4 \pm 11.9$ & $42.5 \pm 19.2$ \\
13 & 0.673291 & +250.3 & $13.93 \pm 0.14$ & $27.6 \pm 10.9$ & $46.4 \pm 18.3$ \\
14 & 0.673562 & +289.9 & $13.38 \pm 0.26$ & $10.5 \pm 6.9$ & $6.7 \pm 4.4$ \\
15 & 0.673787 & +339.1 & $13.68 \pm 0.15$ & $38.4 \pm 14.1$ & $89.9 \pm 33.0$ \\
16 & 0.674297 & +430.5 & $13.11 \pm 0.12$ & $26.1 \pm 10.1$ & $41.6 \pm 16.1$ \\
17 & 0.674872 & +533.5 & $13.70 \pm 0.13$ & $34.6 \pm 8.8$ & $73.0 \pm 18.6$ \\
18 & 0.675272 & +605.1 & $14.26 \pm 0.05$ & $33.7 \pm 4.2$ & $69.4 \pm 8.6$ \\
19 & 0.675789 & +698.8 & $13.82 \pm 0.04$ & $29.0 \pm 3.4$ & $51.5 \pm 5.9$ \\
20 & 0.676366 & +801.2 & $13.10 \pm 0.12$ & $20.5 \pm 7.9$ & $25.7 \pm 9.9$ \\
21 & 0.676776 & +874.7 & $13.86 \pm 0.04$ & $33.5 \pm 3.6$ & $68.3 \pm 7.3$ \\
\hline & & & & &
\end{tabular}

Notes.

${ }^{\text {a }}$ Velocities are measured with respect to $z_{\mathrm{gal}}=0.6719$.

${ }^{\mathrm{b}}$ See the text for discussion of these components, which required a deblending treatment. We also fitted this region with a single VP component at $v=-516.9$, with $\log N=14.88 \pm 0.02$ and $b=32.76 \pm 0.861 \mathrm{~km} \mathrm{~s}^{-1}$. However, the VP model provided a poor match to the structure in the core of the $\operatorname{Ly} \alpha$ feature.

$v \simeq-530 \mathrm{~km} \mathrm{~s}^{-1} \mathrm{Ly} \alpha$ absorption from the H I complex. The resulting deblended profile is illustrated in Figure 4(b).

Prior to and following the deblending process, we experienced difficulty obtaining a satisfactory simultaneous fit to the Ly $\alpha$ and Ly $\beta$ absorption in the $v \simeq-530 \mathrm{~km} \mathrm{~s}^{-1}$ feature. Though the deblending provided an improved VP model, we caution that the number of VP components and the resulting column densities and Doppler $b$ parameters we adopted should be viewed with discretion (see comments for Table 2).

\subsection{Metal Lines}

We examined the COS, STIS, and HIRES quasar spectra for associated metal-line transitions, including the Mg II $\lambda \lambda 2796,2803$, Si IV $\lambda \lambda 1393,1402$, C IV $\lambda \lambda 1548,1550$, and O VI $\lambda \lambda 1031,1037$ zero-volt resonance doublets, and transitions from Si II, Si III, C II, C III, etc. In Figure 5, we present the wavelength regions of the quasar spectra corresponding to detected metal lines C III $\lambda 977$ (COS), C IV $\lambda \lambda 1548,1550$ (STIS), and $\mathrm{O}$ VI $\lambda \lambda 1031,1037$ (COS). We also show the Ly $\alpha$ absorption over the same velocity range. VP components 7-11 are shown, where the "cloud" number is given in Column 1 of Table 2. Only clouds 7,8 , and 10 have detected metals.

In Paper I, we reported no detected metal absorption in $\mathrm{C}$ IV (STIS) and O vi (FOS). However, we clearly detected $\mathrm{O}$ VI absorption in the COS spectrum in the velocity range $-200 \mathrm{~km} \mathrm{~s}^{-1} \leqslant v \leqslant 0 \mathrm{~km} \mathrm{~s}^{-1}$ relative to $z_{\text {gal }}=0.6719$. We also detected C III $\lambda 977$ absorption at $v \simeq-120 \mathrm{~km} \mathrm{~s}^{-1}$. We then re-examined the C IV absorption, and formally detected $\mathrm{C}$ IV $\lambda 1548$ aligned in velocity with the $\mathrm{O}$ VI and $\mathrm{C}$ III absorption. The C IV $\lambda 1550$ absorption, which we had interpreted as noise in Paper I, is detected at the $3.5 \sigma$ level. 

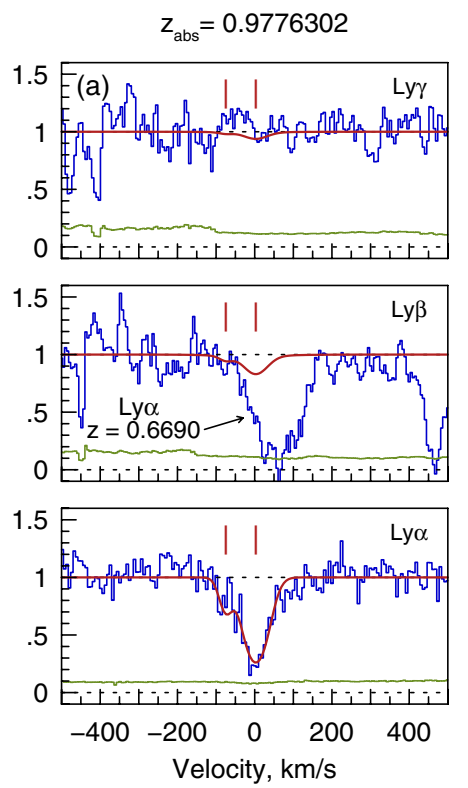

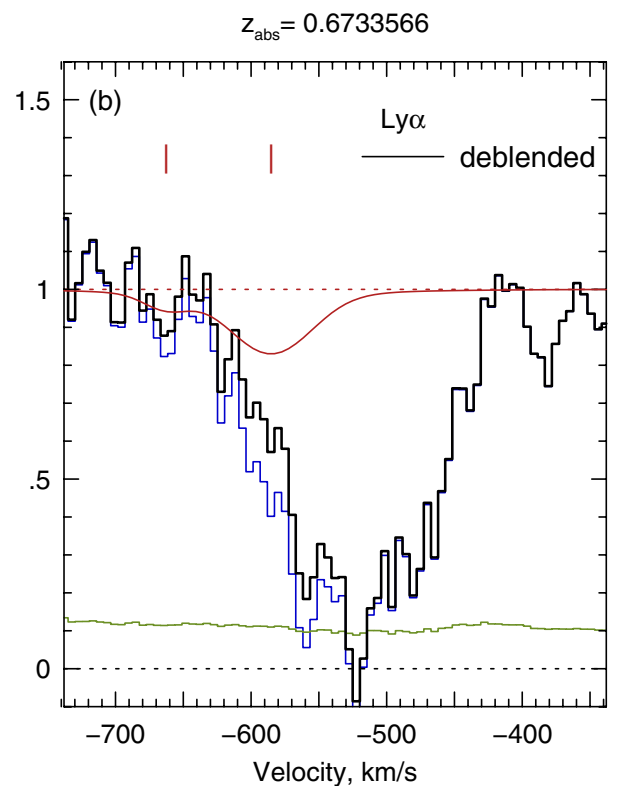

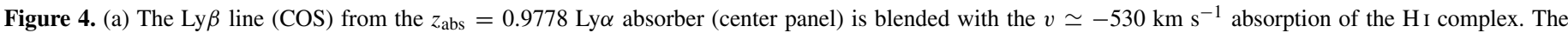

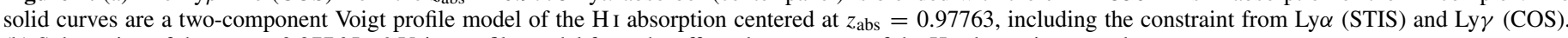
(b) Subtraction of the $z_{\mathrm{abs}}=0.9776 \mathrm{Ly} \beta$ Voigt profile model from the affected component of the H I absorption complex.

(A color version of this figure is available in the online journal.)

In Figure 5, the curves through the data are fitted VP components. For these fits, we modified MINFIT to hold the VP component redshifts (velocities) and Doppler $b$ parameters constant, allowing only the column densities to be $\chi^{2}$ minimized. We fitted the metal lines using the velocities from the VP fits to the H I Lyman series lines and, consistent with the assumption of thermal broadening, scaled the Doppler $b$ parameters for the appropriate ion masses from the VP fits to the H I Lyman series lines. As with the H I fits described above, the COS ISF (Dixon et al. 2011; Kriss 2011) and the STIS ISF (Ely et al. 2011) appropriate for the spectrograph settings and the observed wavelength of each transition were determined via interpolation of the online tabulated data.

When a VP component column density was objectively determined to be insignificant, MINFIT returned an upper limit. Several tests for significance were conducted during the leastsquares fitting convergence; in short, for a column density to be deemed significant, the fitted VP value and its uncertainty had to be inconsistent with the upper limit on the column density (in that precise region of the spectrum and for that Doppler $b$ parameter), and the integrated apparent optical depth (Savage \& Sembach 1991) had to be consistent with the VP component and inconsistent with the upper limit on the column density.

In Table 3, we list the metal-line absorption properties for all clouds. In MINFIT, the equivalent width limits are determined directly from the spectra using the methods of Schneider et al. (1993) and Churchill et al. (1999b), but modified for partially or fully resolved features (see Lawton et al. 2008). For each transition, we use the thermally scaled Doppler $b$ parameter from the VP fit to the H I series convolved with the appropriate ISF to measure the upper limit on the equivalent width in each cloud. The uncertainty in the scaled Doppler $b$ width is used to determine the $1 \sigma$ spread in this limit. The column density limits and the $1 \sigma$ spread in these are determined from the curve of growth.

\section{IONIZATION MODELING}

We employed our own photo+collisional ionization code (C. W. Churchill \& E. Klimek, in preparation), which is very similar to the code LINESPEC (Verner \& Iakovlev 1990). The code treats photoionization, Auger ionization, direct collisional ionization, excitation-autoionization, photo-recombination, highand low-temperature dielectronic recombination, charge transfer ionization by $\mathrm{H}^{+}$, and charge transfer recombination by $\mathrm{H}^{0}$ and $\mathrm{He}^{0}$. Metals up to zinc can be incorporated, and all ionization stages for each elemental species are modeled. The code is appropriate for optically thin gas in which no ionization structure is present.

The input cloud parameters are the hydrogen number density, $n_{\mathrm{H}}$, equilibrium kinetic temperature, $T$, and the mass fraction of metals, $Z$. Solar abundance mass fractions are taken from Table 1.4 of Draine (2011), based upon Asplund et al. (2009), though the code has the option of modifying the relative abundances. A Haardt \& Madau (2012) ionizing spectrum is used for the ultraviolet background (UVB). However, a stellar ionizing spectrum or a combined stellar plus UVB ionizing spectrum can be incorporated (see C. W. Churchill \& E. Klimek, in preparation, for details).

The code obtains an initial guess solution for the density of each ionic species based upon the assumption of adjacent ion stage ionization and recombination balance (i.e., neglecting Auger and charge transfer processes). Then, the rate matrix is solved using the code dqed.f, a Hanson/Krogh nonlinear leastsquares algorithm with linear constraints based on a quadratictensor local model (Hanson 1986). The outputs of the ionization code are the electron density, the ionization and recombination rate coefficients, ionization fractions, and the number densities for all ionic species.

Since the galaxy G1 is $58 \mathrm{kpc}$ from the location where the gas is probed in absorption, and the stellar population is clearly dominated by red stars, we assume a UVB-only ionizing spectrum. We also assume a solar abundance pattern 


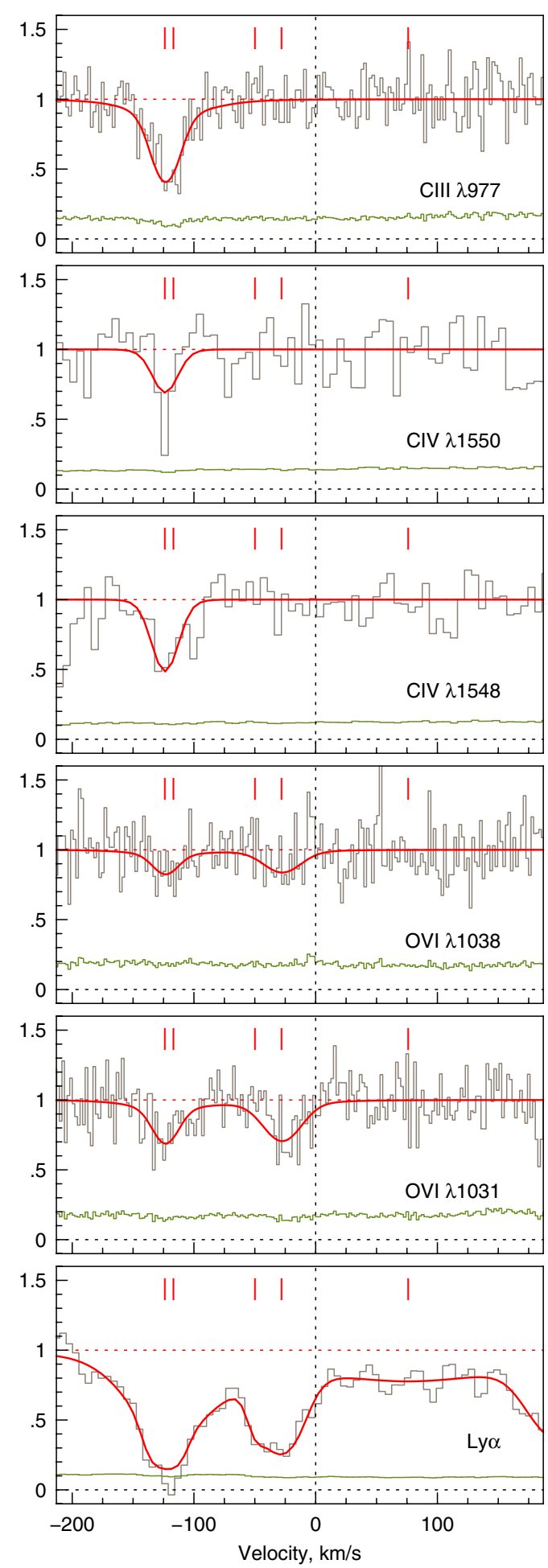

Figure 5. Expanded region of the Voigt profile decomposition of the detected metal lines over the redshift window for clouds 7-11 of the H I complex. The vertical dashed line is the redshift of galaxy G1.

(A color version of this figure is available in the online journal.)

and include metals up to iron (however, we omitted lithium, beryllium, boron, fluorine, and the noble gas elements for which many of the ionization and recombination rates are not well determined).

For a given cloud model, the resulting column density of ionic species $\mathrm{X}$ is obtained by

$$
\log N(\mathrm{X})=\log N\left(\mathrm{H}_{\mathrm{I}}\right)-\log f_{\mathrm{H}_{\mathrm{I}}}+\log n_{\mathrm{X}}-\log n_{\mathrm{H}},
$$

where $N(\mathrm{HI})$ is the $\mathrm{HI}$ column density from the VP fit to the data, $n_{\mathrm{H}}$ is the input hydrogen density of the cloud model, and where $f_{\mathrm{H}_{\mathrm{I}}}$ is the ionization fraction of $\mathrm{H}^{0}$, and $n_{\mathrm{X}}$ is the number density of ionic species $\mathrm{X}$ output by the code, respectively. Note that the metallicity of the model is implied. In practice, $n_{\mathrm{X}}$ in a given model scales directly in proportion to $Z$. Thus, if the metallicity of the cloud model is $Z_{\mathrm{m}}$, for which the resulting density of species $\mathrm{X}$ is $n_{\mathrm{X}, \mathrm{m}}$, then $\log n_{\mathrm{X}}=\log n_{\mathrm{X}, \mathrm{m}}+\left(\log Z-\log Z_{\mathrm{m}}\right)$. For a given $n_{\mathrm{H}}$, such scaling of the models is valid only if $f_{\mathrm{H}_{\mathrm{I}}}$ is independent of metallicity, which holds for $\log Z / Z_{\odot} \leqslant-1$.

Comparing Equation (1) to the measured values of $N(\mathrm{X})$ from our VP fitting, we can constrain the metallicity and $n_{\mathrm{H}}$ of each cloud. The range in these constrained quantities is based upon the $1 \sigma$ uncertainties in the measured column densities. Only three clouds have detected metal lines and therefore measured $N(\mathrm{X})$. These clouds are 7,8 , and 10 . We examine these clouds in the following subsections.

\subsection{Clouds 7 and 8}

Clouds 7 and 8 are located at $v \simeq-120 \mathrm{~km} \mathrm{~s}^{-1}$ with respect to the systemic velocity of $\mathrm{G} 1$ and are separated by $\Delta v=7 \mathrm{~km} \mathrm{~s}^{-1}$, which is on the order of half a single resolution element. The H I profile is highly suggestive of a narrow plus a broad component combining to yield a deep core with a broad wing. The VP components reflect this profile shape, with cloud 7 providing the profile core, $\log N(\mathrm{HI})=14.1$ and $b(\mathrm{HI})=8 \mathrm{~km} \mathrm{~s}^{-1}$, and with cloud 8 providing the broadened wings, $\log N(\mathrm{H} \mathrm{I})=13.7$ and $b(\mathrm{HI})=43 \mathrm{~km} \mathrm{~s}^{-1}$. The requirement for the broader cloud is significant at the $99 \%$ confidence level, even though some contribution to the blue portion of the broad wing is due to the extremely broad VP component (cloud 11) at $v=+76 \mathrm{~km} \mathrm{~s}^{-1}$.

At $v \simeq-120 \mathrm{~km} \mathrm{~s}^{-1}, \mathrm{C}_{\mathrm{III}}, \mathrm{C}_{\mathrm{IV}}$, and $\mathrm{O}$ VI are formally detected in the spectra. However, from the VP fitting, both clouds 7 and 8 have associated $\mathrm{C}$ III and $\mathrm{O}$ vI, whereas only cloud 7 has associated C IV. Inspection of Figure 5 shows that the $\mathrm{C}$ IV absorption appears to be offset in velocity from the $\mathrm{O}$ VI. However, the C IV is measured in the STIS spectrum, whereas the $\mathrm{O}$ VI and $\mathrm{C}$ III are measured in the COS spectrum. Thus, the measured velocity offset relies heavily on the accuracy of the wavelength zero points between the two independent spectra.

Assuming thermal broadening, the implied temperature of cloud 7 is $8000 \mathrm{~K}$, which is too cool to host strong O VI and C IV absorption. The implied temperature of cloud 8 is $T=114,000 \pm 38,000 \mathrm{~K}$, which is consistent with the temperature at which C IV absorption peaks for collisional ionization equilibrium. To analyze this absorption structure and constrain its hydrogen density and metallicity, we opted to assume a single phase of gas that is dominated by cloud 8 (however, in Section 6.5 we also model these clouds as two distinct ionization phases). We added the $\mathrm{H}$ i and metal column densities from clouds 7 and 8 . For this exercise, we found that the column density limits for Mg II, Si III, and Si IV did not contribute to constraining the gas properties.

In Figure 6, we illustrate our analysis of clouds $7+8$. The ionization code was run for $-7 \leqslant \log n_{\mathrm{H}} \leqslant-1$ for $T=$ $114,000 \mathrm{~K}$, and for $T=152,000$ and $T=76,000$, which brackets the $1 \sigma$ uncertainty in the temperature. In Figure 6(a), we illustrate the model column densities for $\mathrm{C}$ III, $\mathrm{C}$ IV, and $\mathrm{O}$ VI for $\log N(\mathrm{HI})=14.3$ and metallicity $\log Z / Z_{\odot}=-2.0$. In Figure $6(\mathrm{~b})$, we show the metallicity constraints as a function of $n_{\mathrm{H}}$ for each metal ion, where the range in the constraints is based on the uncertainty in the temperature and the uncertainty 

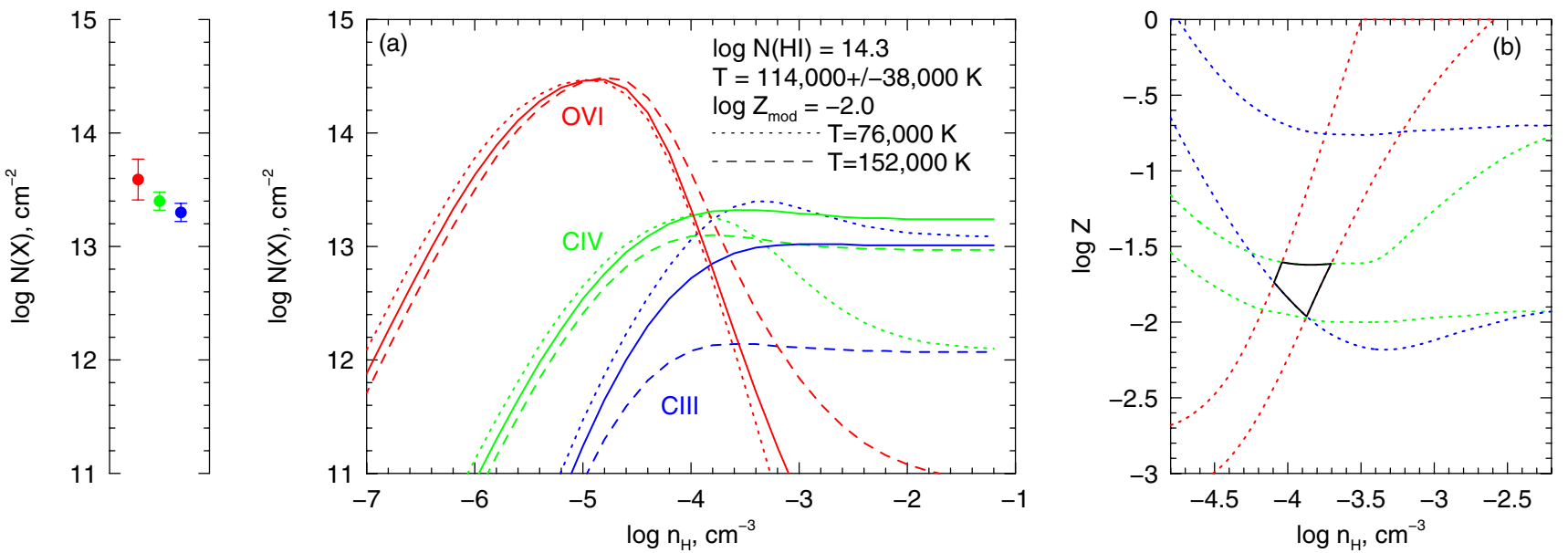

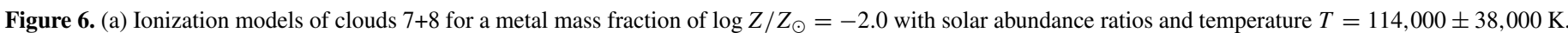

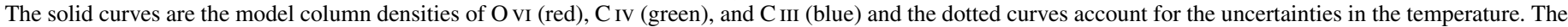

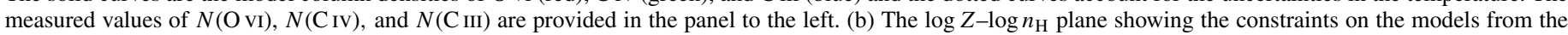
measured data (same color scheme). Solid black curves provide the overlap regions.

(A color version of this figure is available in the online journal.)

Table 3

Selected Metal-line Measurements

\begin{tabular}{|c|c|c|c|c|c|c|c|c|}
\hline Cloud No. & $z_{\mathrm{cl}}$ & $\begin{array}{c}v_{\mathrm{cl}^{\mathrm{a}}} \\
\left(\mathrm{km} \mathrm{s}^{-1}\right)\end{array}$ & $\begin{array}{l}\log N \\
\left(\mathrm{Mg}_{\mathrm{II}}\right)\end{array}$ & $\begin{array}{l}\log N \\
(\mathrm{Si} \text { III) }\end{array}$ & $\begin{array}{l}\log N \\
(\text { Si IV) }\end{array}$ & $\begin{array}{l}\log N \\
(\mathrm{C} \text { III })\end{array}$ & $\begin{array}{l}\log N \\
\left(\mathrm{C}_{\text {IV }}\right)\end{array}$ & $\begin{array}{l}\log N \\
\left(\mathrm{O}_{\mathrm{VI}}\right)\end{array}$ \\
\hline$\overline{1}$ & 0.668645 & -582.1 & $<11.70_{-0.30}^{+0.17}$ & $<12.46_{-9.99}^{+0.16}$ & $<13.07_{-9.99}^{+0.15}$ & $<12.77_{-9.99}^{+0.16}$ & $<13.26_{-9.99}^{+0.16}$ & $<13.58_{-9.99}^{+0.16}$ \\
\hline 2 & 0.668861 & -543.5 & $<11.53_{-0.18}^{+0.13}$ & $<12.29_{-0.16}^{+0.11}$ & $<12.92_{-0.13}^{+0.10}$ & $<12.60_{-0.15}^{+0.11}$ & $<13.12_{-0.16}^{+0.11}$ & $<13.42_{-0.14}^{+0.11}$ \\
\hline 3 & 0.669000 & -518.6 & $<11.50_{-0.25}^{+0.15}$ & $<12.26_{-0.15}^{+0.13}$ & $<12.90_{-9.99}^{+0.12}$ & $<12.57_{-0.06}^{+0.14}$ & $<13.09_{-0.12}^{+0.13}$ & $<13.40_{-0.03}^{+0.13}$ \\
\hline 4 & 0.669112 & -498.5 & $<11.21_{-0.07}^{+0.12}$ & $<12.02_{-9.99}^{+0.08}$ & $<12.79_{-9.99}^{+0.01}$ & $<12.41_{-9.99}^{+0.05}$ & $<12.87_{-9.99}^{+0.08}$ & $<13.24_{-9.99}^{+0.01}$ \\
\hline 5 & 0.669199 & -482.9 & $<11.25_{-0.11}^{+0.09}$ & $<12.05_{-0.06}^{+0.06}$ & $<12.78_{-9.99}^{+0.03}$ & $<12.39_{-9.99}^{+0.04}$ & $<12.90_{-9.99}^{+0.06}$ & $<13.22_{-9.99}^{+0.04}$ \\
\hline 6 & 0.669290 & -520.6 & $<11.54_{-0.20}^{+0.13}$ & $<12.30_{-0.17}^{+0.12}$ & $<12.93_{-0.14}^{+0.11}$ & $<12.61_{-0.16}^{+0.12}$ & $<13.13_{-0.17}^{+0.12}$ & $<13.43_{-0.16}^{+0.12}$ \\
\hline 7 & 0.671203 & -123.8 & $<11.48_{-0.06}^{+0.05}$ & $<12.24_{-0.05}^{+0.04}$ & $<12.89_{-0.04}^{+0.04}$ & $13.24 \pm 0.07$ & $13.40 \pm 0.08$ & $13.49 \pm 0.13$ \\
\hline 8 & 0.671244 & -116.6 & $<11.76_{-0.08}^{+0.07}$ & $<12.53_{-0.07}^{+0.06}$ & $<13.12_{-0.07}^{+0.06}$ & $12.42 \pm 0.39$ & $<13.33_{-0.07}^{+0.06}$ & $12.89 \pm 0.68$ \\
\hline 9 & 0.671617 & -49.6 & $<11.33_{-0.18}^{+0.38}$ & $<12.10_{-0.17}^{+0.31}$ & $<12.81_{-0.16}^{+0.24}$ & $<12.42_{-0.09}^{+0.31}$ & $<12.95_{-0.15}^{+0.31}$ & $<13.25_{-0.11}^{+0.30}$ \\
\hline 10 & 0.671739 & -27.7 & $<11.58_{-0.10}^{+0.08}$ & $<12.33_{-0.09}^{+0.07}$ & $<12.96_{-0.08}^{+0.07}$ & $<12.65_{-0.09}^{+0.07}$ & $<13.16_{-0.09}^{+0.07}$ & $13.62 \pm 0.08$ \\
\hline 11 & 0.672319 & +76.1 & $<11.91_{-0.14}^{+0.11}$ & $<12.72_{-0.13}^{+0.10}$ & $<13.25_{-0.13}^{+0.10}$ & $<12.98_{-0.14}^{+0.10}$ & $<13.47_{-0.14}^{+0.10}$ & $<13.78_{-0.14}^{+0.10}$ \\
\hline 12 & 0.672994 & +197.0 & $<11.65_{-0.11}^{+0.09}$ & $<12.41_{-0.10}^{+0.08}$ & $<13.03_{-0.09}^{+0.07}$ & $<12.73_{-0.10}^{+0.08}$ & $<13.23_{-0.10}^{+0.08}$ & $<13.54_{-0.10}^{+0.08}$ \\
\hline 13 & 0.673291 & +250.3 & $<11.66_{-0.10}^{+0.08}$ & $<12.42_{-0.09}^{+0.07}$ & $<13.04_{-0.08}^{+0.07}$ & $<12.74_{-0.09}^{+0.07}$ & $<13.24_{-0.09}^{+0.07}$ & $<13.55_{-0.09}^{+0.07}$ \\
\hline 14 & 0.673562 & +289.9 & $<11.46_{-0.18}^{+0.12}$ & $<12.22_{-0.14}^{+0.10}$ & $<12.87_{-0.10}^{+0.09}$ & $<12.52_{-0.12}^{+0.11}$ & $<13.05_{-0.14}^{+0.10}$ & $<13.36_{-0.12}^{+0.11}$ \\
\hline 15 & 0.673787 & +339.1 & $<11.73_{-0.09}^{+0.07}$ & $<12.50_{-0.08}^{+0.07}$ & $<13.09_{-0.08}^{+0.06}$ & $<12.81_{-0.08}^{+0.07}$ & $<13.30_{-0.08}^{+0.07}$ & $<13.63_{-0.08}^{+0.07}$ \\
\hline 16 & 0.674297 & +430.5 & $<11.65_{-0.09}^{+0.08}$ & $<12.41_{-0.08}^{+0.07}$ & $<13.03_{-0.08}^{+0.06}$ & $<12.73_{-0.09}^{+0.07}$ & $<13.22_{-0.08}^{+0.07}$ & $<13.54_{-0.08}^{+0.07}$ \\
\hline 17 & 0.674872 & +533.5 & $<11.71_{-0.06}^{+0.05}$ & $<12.47_{-0.05}^{+0.05}$ & $<13.08_{-0.05}^{+0.04}$ & $<12.79_{-0.06}^{+0.05}$ & $<13.28_{-0.05}^{+0.05}$ & $<13.61_{-0.05}^{+0.05}$ \\
\hline 18 & 0.675272 & +605.1 & $<11.71_{-0.03}^{+0.03}$ & $<12.47_{-0.03}^{+0.02}$ & $<13.07_{-0.02}^{+0.02}$ & $<12.78_{-0.03}^{+0.02}$ & $<13.28_{-0.03}^{+0.02}$ & $<13.60_{-0.03}^{+0.02}$ \\
\hline 19 & 0.675789 & +698.8 & $<11.67_{-0.03}^{+0.02}$ & $<12.43_{-0.02}^{+0.02}$ & $<13.05_{-0.02}^{+0.02}$ & $<12.75_{-0.02}^{+0.02}$ & $<13.25_{-0.02}^{+0.02}$ & $<13.56_{-0.02}^{+0.02}$ \\
\hline 20 & 0.676366 & +801.2 & $<11.60_{-0.09}^{+0.08}$ & $<12.35_{-0.08}^{+0.07}$ & $<12.99_{-0.07}^{+0.06}$ & $<12.67_{-0.09}^{+0.07}$ & $<13.18_{-0.08}^{+0.07}$ & $<13.48_{-0.08}^{+0.07}$ \\
\hline 21 & 0.676776 & +874.7 & $<11.70_{-0.02}^{+0.02}$ & $<12.46_{-0.02}^{+0.02}$ & $<13.07_{-0.02}^{+0.02}$ & $<12.78_{-0.02}^{+0.02}$ & $<13.27_{-0.02}^{+0.02}$ & $<13.60_{-0.02}^{+0.02}$ \\
\hline
\end{tabular}

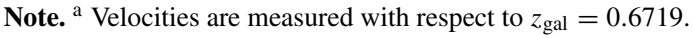

in the $\mathrm{H}$ I and metal ion column densities. The region of overlap indicates where the three ions provide consistent constraints on $n_{\mathrm{H}}$ and $\log Z / Z_{\odot}$. Allowing for the uncertainties in the data, the gas is constrained to have $-4.2 \leqslant \log n_{\mathrm{H}} \leqslant-3.7$ and $-2.0 \leqslant \log Z / Z_{\odot} \leqslant-1.6$ by all three metal ions. Note that metallicity is constrained primarily by $N(\mathrm{C}$ III), whereas hydrogen density is constrained primarily by $N(\mathrm{O}$ vI).

\subsection{Cloud 10}

Cloud 10 is located at $v \simeq-30 \mathrm{~km} \mathrm{~s}^{-1}$ with respect to the systemic velocity of G1. It is optically thin in neutral hydrogen, with $\log N(\mathrm{HI})=13.7 \pm 0.2$ and an implied temperature of $T=20,500 \pm 8600 \mathrm{~K}$. The only detected metal line absorption is from $\mathrm{O}$ VI, with $\log N(\mathrm{O}$ VI $)=13.6 \pm 0.1$.

A high abundance of the $\mathrm{O}^{+5}$ ion in a $T=20,000 \mathrm{~K}$ cloud may be somewhat surprising. For $n_{\mathrm{H}} \leqslant-3$ and $\log Z / Z_{\odot}=-2$, analysis of the rate coefficients output by the ionization code shows that the balance of $\mathrm{O}^{+4}, \mathrm{O}^{+5}$, and $\mathrm{O}^{+6}$ is dominated by photoionization and recombination via charge exchange with $\mathrm{H}^{+}$, which dominates over free electron recombination by two orders of magnitude for $\mathrm{O}^{+5}$.

For cloud 10, the measured upper limit on $\mathrm{CIII}$ is $\log N\left(\mathrm{C}_{\text {III }}\right) \leqslant 12.7$ and on $\mathrm{C}_{\mathrm{IV}}$ is $\log N(\mathrm{C}$ IV $) \leqslant 13.2$. As 

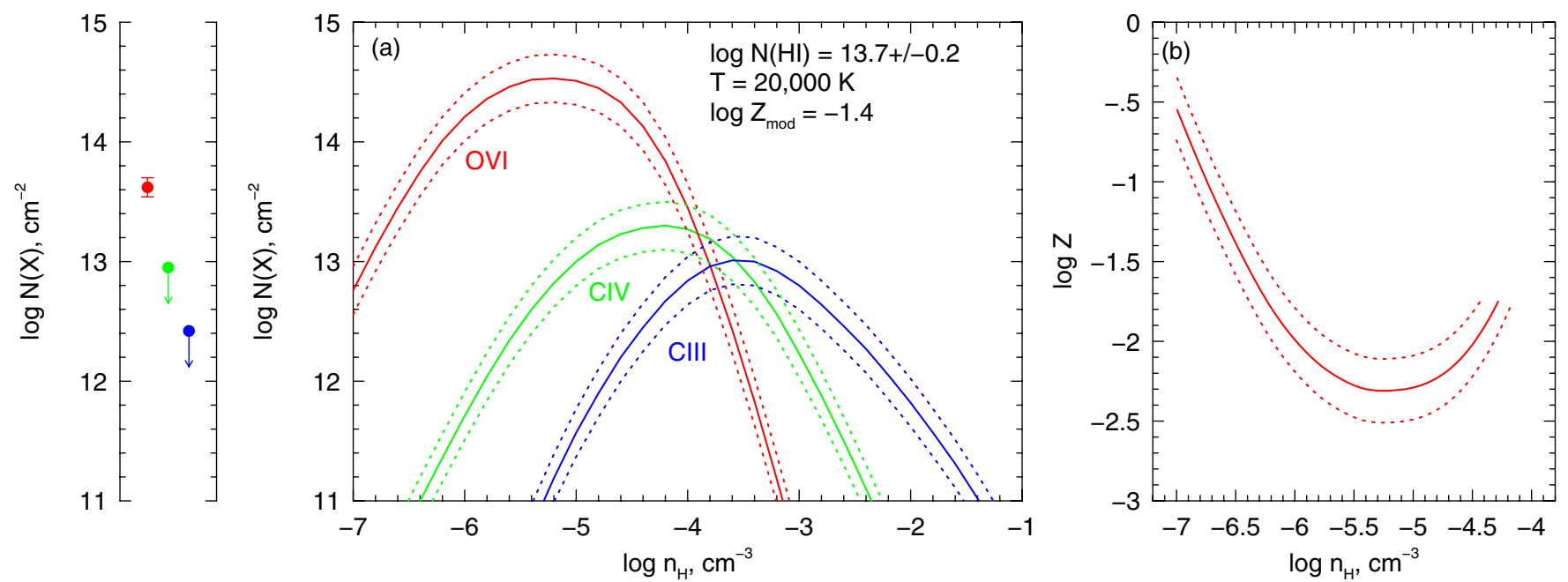

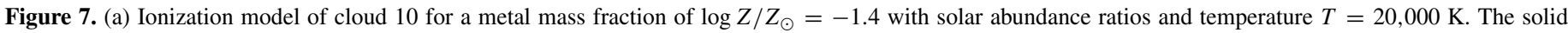

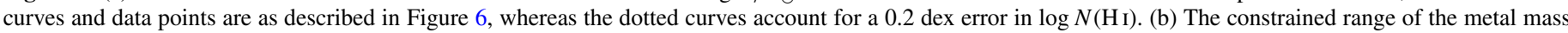
fraction on the $\log Z-\log n_{\mathrm{H}}$ plane, accounting for the column density limits on the carbon ions.

(A color version of this figure is available in the online journal.)

with our exercise for clouds $7+8$, we found that the column density limits for Mg II, Si III, and Si IV did not contribute to constraining the gas properties.

In Figure 7, we illustrate our analysis of cloud 10. The ionization code was run for $-7 \leqslant \log n_{\mathrm{H}} \leqslant-1$ for $T=$ $20,000 \mathrm{~K}$. In Figure 7(a), we show the column densities for $\mathrm{C}$ III, C IV, and $\mathrm{O}$ VI for $\log N(\mathrm{HI})=13.7 \pm 0.2$ and metallicity $\log Z / Z_{\odot}=-1.4$. The metallicity and hydrogen density constraints for cloud 10 are shown in Figure 7(b).

If we account for the uncertainty in the implied temperature, we find that for $T=28,000 \mathrm{~K}$, the curves in Figure 7(b) are unchanged for $\log n_{\mathrm{H}} \geqslant-5.3$, and are shifted upward in the diagram by no more than 0.7 dex (higher $Z$ at a given $\left.n_{\mathrm{H}}\right)$ for $\log n_{\mathrm{H}}<-5.3$. As the temperature is decreased, the curves shift to the upper right in the diagram such that at $T=12,000 \mathrm{~K}$, a minimum metallicity in the allowed range $-2.3 \leqslant \log Z / Z_{\odot} \leqslant-1.8$ occurs at $\log n_{\mathrm{H}} \geqslant-3.7$ instead of $-2.5 \leqslant \log Z / Z_{\odot} \leqslant-2.0$ at $\log n_{\mathrm{H}}=-5.3$. It seems less likely that $\mathrm{O}$ VI absorption would be as strong as we detect in $12,000 \mathrm{~K}$ gas.

\subsection{Clouds with No Detected Metal Lines}

The remainder of the $\mathrm{HI}$ absorption complex is characterized by multiple components with gas temperatures in the range 20,000-90,000 K, though a hot, nearly million degree component is also present (cloud 11). For the clouds with no detected metals, the densities, ionization corrections, and therefore metallicities cannot be constrained without additional assumptions. In Section 6.5, we assume hydrodynamic equilibrium and undertake an analysis of the clouds with only upper limits on the metal line column densities. Below, we motivate the assumption of hydrodynamic equilibrium as a reasonable scenario.

\section{INFERRED PHYSICAL CONDITIONS OF THE GAS}

\subsection{Ionization Equilibrium and Cloud Stability}

In general, ionization equilibrium is valid when the collisional ionization timescale, $\tau_{\text {coll }}$, and the photoionization timescale, $\tau_{\mathrm{ph}}$, are shorter than the cooling time of the gas, $\tau_{\mathrm{cool}}$.
For a monatomic gas, the cooling time is the ratio of the energy per unit volume and the energy loss per unit volume per unit time, $\tau_{\text {cool }}=Q / \dot{Q}=3 / 2\left(n_{\mathrm{e}}+n_{\mathrm{A}}\right) k T / n_{\mathrm{e}} n_{\mathrm{A}} \Lambda(T, Z)$, where $n_{\mathrm{e}}$ is the electron density, $n_{\mathrm{A}}$ is the total density of all ions, and $\Lambda(T, Z)$ is the specific cooling function for metallicity $Z$. We obtain $n_{\mathrm{e}}$ and $n_{\mathrm{A}}$ from our ionization code as a function of $n_{\mathrm{H}}$ and $T$. Our cloud models are optically thin, such that $n_{\mathrm{A}} \propto n_{\mathrm{H}}$ and $n_{\mathrm{e}} \propto n_{\mathrm{H}}$ at a given $T$; we thus find that the behavior of the cooling time follows $\tau_{\text {cool }} \propto n_{\mathrm{H}}^{-1}$ at fixed $T$.

We adopt the $Z=0$ specific cooling function of Sutherland \& Dopita (1993) for a gas in collisional ionization equilibrium (also see Dopita \& Sutherland 2003). Since our models include photoionization, we corrected for photoionization heating (see Osterbrock \& Ferland 2006), which reduces the magnitude of $\dot{Q}$, and therefore increases the estimated cooling time. We found that photoionization heating from the UVB at $z=0.67$ is negligible for the density and temperatures ranges we explored.

Metals strongly contribute to the cooling rate, so the $Z=0$ curve provides an upper limit on the estimated cooling time under the assumption of ionization equilibrium. However, the effect is negligible for $\log Z / Z_{\odot}=-3$ and is a maximum difference of 0.4 dex at $\log T \simeq 5.3$ for $\log Z / Z_{\odot}=-2$ (see Sutherland \& Dopita 1993).

For a given ion, the collisional timescale is well approximated as $\tau_{\text {coll }} \simeq 1 /\left[n_{\mathrm{e}}\left(\alpha_{\text {rec }}+\alpha_{\text {coll }}\right)\right]$, whereas the photoionization timescale is $\tau_{\mathrm{ph}} \simeq 1 / R_{\mathrm{ph}}\left(J_{v}\right)$, where $\alpha_{\text {rec }}(T)$ and $\alpha_{\text {coll }}(T)$ are the recombination and collisional ionization rate coefficients, and $R_{\text {ph }}\left(J_{v}\right)$ is the photoionization rate for the given ion, respectively. We obtain these quantities directly from our ionization code. Since $n_{\mathrm{e}} \propto n_{\mathrm{H}}$ at a given $T$, we have $\tau_{\text {coll }} \propto n_{\mathrm{H}}^{-1}$ at fixed $T$. Thus, the ratio $\tau_{\text {cool }} / \tau_{\text {coll }}$ is effectively constant as a function of $n_{\mathrm{H}}$ for fixed temperature. Note that for lower temperatures, $\alpha_{\text {coll }}(T)$ vanishes so that $\tau_{\text {coll }}$ becomes equal to the recombination timescale, $\tau_{\text {rec }} \simeq 1 / n_{\mathrm{e}} \alpha_{\text {rec }}$.

\subsection{Equilibrium Based on Hydrogen}

In what follows, we briefly explore selected clouds using representative $n_{\mathrm{H}}$ densities for the purpose of illustration. In Table 4, we present a summary of cloud models for the $n_{\mathrm{H}}$ values 
Table 4

Cloud Models of the H I Complex ${ }^{\mathrm{a}}$

\begin{tabular}{|c|c|c|c|c|c|c|c|c|c|c|c|c|c|c|c|}
\hline Cloud No. & $\begin{array}{l}N(\mathrm{HI}) \\
\left(\mathrm{cm}^{-2}\right)\end{array}$ & $\begin{array}{c}T \\
(\mathrm{~K})\end{array}$ & $\begin{array}{c}n_{\mathrm{H}} \\
\left(\mathrm{cm}^{-3}\right)\end{array}$ & $f_{\mathrm{H}_{\mathrm{I}}}$ & $\begin{array}{c}\alpha_{\mathrm{rec}} \\
\left(\mathrm{cm}^{3} \mathrm{~s}^{-1}\right)\end{array}$ & $\begin{array}{c}\alpha_{\text {coll }} \\
\left(\mathrm{cm}^{3} \mathrm{~s}^{-1}\right)\end{array}$ & $\begin{array}{c}\tau_{\mathrm{cool}} \\
(\mathrm{yr})\end{array}$ & $\begin{array}{l}\tau_{\text {coll }} \\
(\mathrm{yr})\end{array}$ & $\begin{array}{l}\tau_{\text {dyn }} \\
(\mathrm{yr})\end{array}$ & $\begin{array}{c}\tau_{\mathrm{sc}} \\
(\mathrm{yr})\end{array}$ & $\begin{array}{c}L \\
(\mathrm{kpc})\end{array}$ & $\begin{array}{c}M_{g} \\
\left(M_{\odot}\right)\end{array}$ & $\begin{array}{c}L_{J} \\
(\mathrm{kpc})\end{array}$ & $\begin{array}{c}M_{J} \\
\left(M_{\odot}\right)\end{array}$ & Note $^{b}$ \\
\hline $7+8$ & 14.30 & 5.06 & -5.0 & -6.62 & -13.21 & -8.28 & 9.40 & 5.70 & 10.01 & 11.71 & 4.43 & 15.84 & 2.73 & 10.72 & $\mathrm{JU}$ \\
\hline $7+8$ & 14.30 & 5.06 & -4.0 & -5.69 & -13.21 & -8.28 & 8.40 & 4.70 & 9.51 & 9.78 & 2.51 & 11.05 & 2.23 & 10.22 & $\mathrm{JU}$ \\
\hline $7+8$ & 14.30 & 5.06 & -3.0 & -5.10 & -13.21 & -8.28 & 7.40 & 3.70 & 9.01 & 8.19 & 0.91 & 7.27 & 1.73 & 9.72 & $\mathrm{EE}$ \\
\hline 10 & 13.70 & 4.31 & -5.0 & -6.02 & -12.62 & -11.36 & 8.13 & 8.75 & 10.01 & 10.88 & 3.23 & 12.22 & 2.36 & 9.60 & $\mathrm{JU}$ \\
\hline 10 & 13.70 & 4.31 & -4.0 & -5.02 & -12.62 & -11.36 & 7.13 & 7.76 & 9.51 & 8.88 & 1.23 & 7.22 & 1.86 & 9.10 & $\mathrm{EE}$ \\
\hline 10 & 13.70 & 4.31 & -3.0 & -4.03 & -12.62 & -11.36 & 6.14 & 6.77 & 9.01 & 6.89 & -0.76 & 2.25 & 1.36 & 8.60 & $\mathrm{EE}$ \\
\hline 11 & 13.43 & 5.68 & -5.0 & -7.22 & -13.78 & -7.57 & 10.86 & 4.99 & 10.01 & 11.13 & 4.17 & 15.03 & 3.04 & 11.65 & JU \\
\hline 11 & 13.43 & 5.68 & -4.0 & -6.48 & -13.78 & -7.57 & 9.86 & 3.99 & 9.51 & 9.39 & 2.43 & 10.81 & 2.54 & 11.15 & $\mathrm{EE}$ \\
\hline 11 & 13.43 & 5.68 & -3.0 & -6.23 & -13.78 & -7.57 & 8.86 & 2.99 & 9.01 & 8.14 & 1.17 & 8.04 & 2.04 & 10.65 & EE \\
\hline 15 & 13.68 & 4.95 & -5.0 & -6.53 & -13.12 & -8.50 & 9.06 & 5.92 & 10.01 & 11.05 & 3.72 & 13.69 & 2.68 & 10.56 & JU \\
\hline 15 & 13.68 & 4.95 & -4.0 & -5.58 & -13.12 & -8.50 & 8.06 & 4.92 & 9.51 & 9.10 & 1.77 & 8.84 & 2.18 & 10.06 & $\mathrm{EE}$ \\
\hline 15 & 13.68 & 4.95 & -3.0 & -4.88 & -13.12 & -8.50 & 7.06 & 3.93 & 9.01 & 7.40 & 0.07 & 4.73 & 1.68 & 9.56 & $\mathrm{EE}$ \\
\hline 18 & 14.26 & 4.84 & -5.0 & -6.43 & -13.03 & -8.78 & 9.06 & 6.20 & 10.01 & 11.59 & 4.20 & 15.14 & 2.62 & 10.40 & JU \\
\hline 18 & 14.26 & 4.84 & -4.0 & -5.46 & -13.03 & -8.78 & 8.06 & 5.20 & 9.51 & 9.62 & 2.23 & 10.23 & 2.12 & 9.90 & $\mathrm{JU}$ \\
\hline 18 & 14.26 & 4.84 & -3.0 & -4.66 & -13.03 & -8.78 & 7.06 & 4.21 & 9.01 & 7.82 & 0.43 & 5.82 & 1.62 & 9.40 & $\mathrm{EE}$ \\
\hline 20 & 13.10 & 4.41 & -5.0 & -6.09 & -12.69 & -10.64 & 8.41 & 8.05 & 10.01 & 10.30 & 2.70 & 10.63 & 2.41 & 9.75 & JU \\
\hline 20 & 13.10 & 4.41 & -4.0 & -5.09 & -12.69 & -10.64 & 7.41 & 7.05 & 9.51 & 8.30 & 0.70 & 5.63 & 1.91 & 9.25 & EE \\
\hline 20 & 13.10 & 4.41 & -3.0 & -4.11 & -12.69 & -10.64 & 6.41 & 6.07 & 9.01 & 6.32 & -1.28 & 0.69 & 1.41 & 8.75 & $\mathrm{EE}$ \\
\hline
\end{tabular}

Notes.

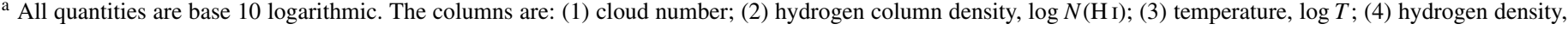

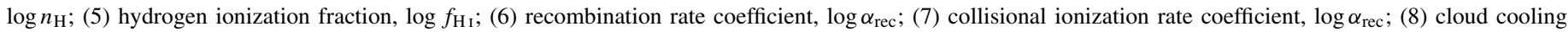

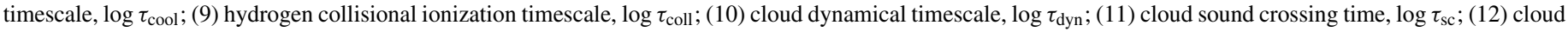
absorption length scale, $\log L ;(13)$ cloud gas mass, $\log M_{g} ;(14)$ cloud Jeans length, $\log L_{J} ;$ and (15) cloud Jeans gas mass, log $M_{J}$.

b JU denotes that the cloud is Jeans unstable or moderately Jeans unstable; EE denotes instability or slight instability toward expansion and/or evaporation.

$\log n_{\mathrm{H}}=-5,-4$, and -3 , where ion specific quantities apply for hydrogen. The hydrogen photoionization rate from the UVB at $z=0.67$ is $R_{\mathrm{ph}}=3.0 \times 10^{-12} \mathrm{~s}^{-1}$, yielding $\tau_{\mathrm{ph}}=1 \times 10^{4}$ yr. As we shall see, the photoionization time is significantly shorter than the cooling times for all models; as such, only the longer collisional timescales constrain whether the clouds are in ionization equilibrium.

For clouds 7+8, at $T=114,000 \mathrm{~K}, \alpha_{\text {rec }}=6.2 \times 10^{-14}$ and $\alpha_{\text {coll }}=5.3 \times 10^{-9} \mathrm{~cm}^{3} \mathrm{~s}^{-1}$, respectively. Assuming $\log n_{\mathrm{H}}=$ -4 , we obtain $\tau_{\text {cool }} \simeq 3 \times 10^{8}$ yr and $\tau_{\text {coll }}=5 \times 10^{4}$ yr. With $\tau_{\text {cool }} / \tau_{\text {coll }} \simeq 5 \times 10^{3}$, hydrogen is clearly in ionization equilibrium in clouds $7+8$; the short photoionization and collisional ionization timescales relative to the recombination timescale are indicative of the highly ionized condition $\left(f_{\mathrm{H}_{\mathrm{I}}}=2 \times 10^{-6}\right)$. The condition $\tau_{\mathrm{ph}} \lesssim \tau_{\text {coll }}$ indicates photoionization marginally dominates over collisional ionization.

For cloud 10, at $T=20,000 \mathrm{~K}, \alpha_{\text {rec }}=2.4 \times 10^{-13}$ and $\alpha_{\text {coll }}=4.3 \times 10^{-12} \mathrm{~cm}^{3} \mathrm{~s}^{-1}$. This cloud is constrained to have $\log n_{\mathrm{H}}<-4$; assuming $\log n_{\mathrm{H}}=-5$, we obtain $\tau_{\text {cool }} \simeq 1 \times 10^{8}$ $\mathrm{yr}$ and $\tau_{\text {coll }}=6 \times 10^{8} \mathrm{yr}$. For this assumed density, we find $\tau_{\text {cool }} / \tau_{\text {coll }} \simeq 0.2$, suggesting that ionization equilibrium may be marginal in cloud 10 for hydrogen (the gas temperature evolves on a similar timescale that the balance can be achieved). As listed in Table 4, the thermal timescale is shorter than the ionization timescale for the range of $n_{\mathrm{H}}$ presented.

For cloud 11, the hot, $\log T=5.68 \mathrm{~K}$, cloud, we have $\alpha_{\text {rec }}=1.7 \times 10^{-14}$ and $\alpha_{\text {coll }}=2.7 \times 10^{-8} \mathrm{~cm}^{3} \mathrm{~s}^{-1}$. For $\log n_{\mathrm{H}}=-4$ and -5 , we obtain $\tau_{\text {cool }} \simeq 7 \times 10^{9-10} \mathrm{yr}$, and $\tau_{\text {coll }}=1 \times 10^{4-5} \mathrm{yr}$, where the longer times correspond to the lower density. Cloud 11 has $\tau_{\text {cool }} / \tau_{\text {coll }} \simeq 7 \times 10^{5}$, indicating an ionization equilibrium condition. Note that if $\log n_{\mathrm{H}}=-4$, we have $\tau_{\mathrm{ph}} \simeq \tau_{\text {coll }}$, indicating that hydrogen ionization $\left(f_{\mathrm{H}_{\mathrm{I}}}=3 \times 10^{-7}\right)$ is driven by both photo and collisional processes. If $\log n_{\mathrm{H}}=-5$, then $f_{\mathrm{HI}}=6 \times 10^{-8}$, which is dominated by photoionization $\left(\tau_{\text {coll }} / \tau_{\mathrm{ph}}=10\right)$; the larger ratio due to the longer recombination timescale results in a higher ionization condition for this lower density.

With regard to the remaining clouds in the H I complex with only limits on the metal line measurements, we selected clouds $15\left(\log N\left(\mathrm{H}_{\mathrm{I}}\right)=13.7, \log T=4.95\right), 18(\log N(\mathrm{H} \mathrm{I})=$ $14.3, \log T=4.84)$, and $20(\log N(\mathrm{HI})=13.1, \log T=4.41)$ as representative. Of clouds with no detected metals, 15 has the highest temperature and an intermediate $N(\mathrm{HI})$ as compared to all clouds in the H I complex. Cloud 20 has the lowest temperature and the smallest $N(\mathrm{HI})$, and cloud 18 has an intermediate temperature and the highest $N(\mathrm{HI})$. The models, for hydrogen, are given in Table 4 . Note that $\tau_{\text {cool }}>\tau_{\text {coll }}$ for these representative clouds, indicating the validity of ionization equilibrium.

\subsection{Equilibrium Based on $\mathrm{O}^{+5}$ and $\mathrm{C}^{+3}$}

For strict ionization equilibrium to hold, the ionization timescales must be smaller than the cooling time for all ions. The same arguments invoked for hydrogen above apply to the metals. Consider the $\mathrm{O}^{+5}$ ion; the photoionization rate from the UVB at $z=0.67$ is $R_{\mathrm{ph}}=4.3 \times 10^{-16} \mathrm{~s}^{-1}$, which yields $\tau_{\mathrm{ph}}=7 \times 10^{7} \mathrm{yr}$.

For clouds $7+8$, at $T=114,000 \mathrm{~K}, \alpha_{\text {rec }}$ and $\alpha_{\text {coll }}$ are $3.7 \times 10^{-12}$ and $3.8 \times 10^{-16} \mathrm{~cm}^{3} \mathrm{~s}^{-1}$, respectively. For $\log n_{\mathrm{H}}=$ -4, we obtain $\tau_{\text {coll }}=\tau_{\text {rec }}=7 \times 10^{7} \mathrm{yr}$, where both scale in proportion to $n_{\mathrm{H}}^{-1}$. We thus have $\tau_{\text {coll }}=\tau_{\text {rec }} \simeq 4 \tau_{\text {cool }}$ independent of $n_{\mathrm{H}}$, indicating that the $\mathrm{O}^{+5}$ ion is marginally in ionization equilibrium. Similarly, we find $\tau_{\text {coll }} \simeq 3 \tau_{\text {cool }}$ for $\mathrm{C}^{+3}$.

For cloud 10, at $T=20,000 \mathrm{~K}, \alpha_{\text {rec }}=1.4 \times 10^{-11} \mathrm{~cm}^{3} \mathrm{~s}^{-1}$ and $\alpha_{\text {coll }}$ is negligible, indicating that collisional processes have vanishing importance in the ionization balance of $\mathrm{O}^{+5}$. 
For $\log n_{\mathrm{H}}=-5$, we obtain $\tau_{\text {coll }}=\tau_{\text {rec }}=2 \times 10^{8}$ yr. For all densities, we have $\tau_{\text {cool }} / \tau_{\text {coll }} \simeq 0$.8. Similarly, for $\mathrm{C}^{+3}$, we find $\tau_{\text {cool }} / \tau_{\text {coll }} \simeq 0.3$. As with hydrogen, ionization equilibrium may be marginal for cloud 10 for $\mathrm{O}^{+5}$ and $\mathrm{C}^{+3}$.

For cloud 11, at $\log T=5.68$, we have $\alpha_{\text {rec }}=1.2 \times 10^{-12}$ and $\alpha_{\text {coll }}=6.9 \times 10^{-11} \mathrm{~cm}^{3} \mathrm{~s}^{-1}$ for $\mathrm{O}^{+5}$. When $\log n_{\mathrm{H}}=-4$, and -5 , we obtain $\tau_{\text {coll }}=4 \times 10^{6-7} \mathrm{yr}$, where the longer times correspond to the lower density. We have $\tau_{\text {cool }} / \tau_{\text {coll }} \simeq 2000$ for which $\mathrm{O}^{+5}$ is predominantly in collisional equilibrium, i.e., $\tau_{\mathrm{coll}} / \tau_{\mathrm{ph}} \simeq 20$ if $\log n_{\mathrm{H}}=-4$, and roughly equally balanced by photo and collisional equilibrium, i.e., $\tau_{\text {coll }} \simeq \tau_{\mathrm{ph}}$ for $\log n_{\mathrm{H}}=-5$. For $\mathrm{C}^{+3}$, we obtain $\tau_{\text {cool }} / \tau_{\text {coll }} \simeq 2 \times 10^{4}$. Cloud 11 is clearly in ionization equilibrium.

For clouds 15,18 , and 20 , we find $\tau_{\text {cool }} / \tau_{\text {coll }} \simeq 2,2$, and 1 , respectively, for $\mathrm{O}^{+5}$. For $\mathrm{C}^{+3}$, we find $\tau_{\text {cool }} / \tau_{\text {coll }} \simeq 0.9$, 0.9 , and 0.4 , respectively. For these clouds, $\mathrm{O}^{+5}$ is marginally in ionization equilibrium, whereas $\mathrm{C}^{+3}$ is marginally not in equilibrium.

Note that the cooling times are 1-3 orders of magnitude shorter than the Hubble time, $\tau_{\mathrm{H}}=1 / H_{0}=1.4 \times 10^{10} \mathrm{yr}$, except for cloud 11 for $\log n_{\mathrm{H}} \leqslant-5$ (recall that the cooling time scales inversely with $n_{\mathrm{H}}$ for fixed $T$ ). This would indicate that the cloud thermal conditions have evolved; however, it is not possible to estimate the thermal histories of the clouds beyond speculating that they were hotter (and presumably less dense) at $z>0.67$. For $4 \leqslant \log T \leqslant 4.7$ and for $5 \leqslant \log T \leqslant 6$, the cooling time increases toward higher temperatures, so we can infer that the thermal evolution of clouds in these temperature was slower at epochs prior to $z=0.67$ assuming the cloud densities have not strongly evolved.

\subsection{Dynamical Stability, Sizes, and Masses}

In view of the inferred thermal evolution of the absorbing clouds, it is interesting to examine their dynamical stability. This can be achieved by comparing the cloud dynamical and sound crossing timescales. Additionally, it would be of interest to estimate the cloud sizes and masses.

To within a dimensionless factor of order unity, the dynamical time (see Lequeux 2005, Section 14.2.1), or free-fall time, of a cloud with total mass density $\rho=\rho_{g} / f_{g}=\left(A_{\mathrm{H}} m_{\mathrm{a}} / x_{\mathrm{H}} f_{g}\right) n_{\mathrm{H}}$, is $\tau_{\text {dyn }}=1 / \sqrt{G \rho}$, where $A_{\mathrm{H}}$ is the atomic weight of hydrogen, $m_{\mathrm{a}}$ is the atomic mass unit, $x_{\mathrm{H}}=0.72$ is the mass fraction of hydrogen ${ }^{12}$ and $f_{g}=\Omega_{b} / \Omega_{m} \simeq 0.16$ is the fraction of the baryonic mass in gas. Evaluating, we have $\tau_{\text {dyn }} \simeq$ $3.2 \times 10^{7} n_{\mathrm{H}}{ }^{-1 / 2} \mathrm{yr}$.

The sound crossing time is $\tau_{\mathrm{sc}}=L / c_{s}$, where $L$ is the physical length scale of the cloud and the sound speed is $c_{s}^{2}=\gamma P_{g} / \rho_{g}=$ $\gamma k T / \mu m_{\mathrm{a}}$, where $P_{g}$ is the gas pressure, $\gamma=5 / 3$ for an ideal monatomic gas, and $\mu$ is the mean molecular weight. Our ionization models indicate that the clouds are highly ionized, so we assume $\mu=\left(2 x_{\mathrm{H}}+3 / 4 x_{\mathrm{He}}+1 / 2 x_{\mathrm{Z}}\right)^{-1} \simeq 0.6$, appropriate for a fully ionized gas with $\log Z / Z_{\odot} \simeq-2$. We thus obtain $\tau_{\mathrm{sc}} \simeq 6.4 \times 10^{9} L T^{-1 / 2} \mathrm{yr}$, when $L$ is given in kpc.

The "absorption" length scale of the cloud can be estimated as the line-of-sight path length required to give rise to the measured $N(\mathrm{HI})$ for the inferred $n_{\mathrm{H}}$ from the ionization models, $L=N(\mathrm{HI}) /\left(f_{\mathrm{H}_{\mathrm{I}}} n_{\mathrm{H}}\right)$. Assuming spherical geometry, the gas mass of the cloud is crudely estimated from $M_{g}=\rho_{g} L^{3} \simeq$ $3.4 \times 10^{7} n_{\mathrm{H}} L^{3} M_{\odot}$ when $L$ is given in kpc. A spherical geometry

\footnotetext{
12 We have assumed a solar helium to hydrogen abundance ratio and solar metal abundance ratios for $\log Z / Z_{\odot}=-2$. A low-metallicity primordial abundance pattern has $x_{\mathrm{H}} \simeq 0.76$, which yields a $5 \%$ difference in $\rho_{g}$.
}

with volume filling factor of unity is likely a very poor model, and one which will significantly overestimate the cloud mass. If the absorber geometry is cylindrical of length $L$ and radius $R$ with aspect ratio $\beta=2 R / L$, then $M_{\text {cyl }} / M_{\text {sph }} \propto \beta^{2}$ if the line of sight probes parallel to $L$.

It is well established based on observational and theoretical grounds that, in general, Ly $\alpha$ forest clouds cannot be pressure confined (Rauch 1998). Schaye (2001) convincingly argues that clouds which develop due to the gravitational influence of underlying dark matter density perturbations persist in a near hydrodynamic equilibrium state local to the region giving rise to the Ly a absorption. Effectively, this is equivalent to stating that the dynamical time is equal to the sound crossing time, $\tau_{\text {dyn }} \simeq \tau_{\text {sc }}$, consequently implying that the characteristic length of the absorbing region will be on the order of the local Jeans length, $L_{J}$ (see Lequeux 2005, Section 14.1.2). Equating $\tau_{\text {dyn }}$ and $\tau_{\mathrm{sc}}$, the Jeans length is $L_{J} \simeq 5.0 \times 10^{-3}\left(T / n_{\mathrm{H}}\right)^{1 / 2} \mathrm{kpc}$, from which the Jeans gas mass ${ }^{13}$ can be estimated, $M_{J}=\rho_{g} L_{J}^{3} \simeq$ $4.3 T^{3 / 2} n_{\mathrm{H}}{ }^{-1 / 2} M_{\odot}$.

For $M_{g}>M_{J}$ the condition is $\tau_{\mathrm{dyn}}<\tau_{\mathrm{sc}}$; the cloud is Jeans unstable to further gravitational contraction and will adjust on the dynamical timescale via fragmentation or due to shock processes. Conversely, for $M_{g}<M_{J}$ the condition is $\tau_{\mathrm{sc}}<\tau_{\mathrm{dyn}}$, and the cloud will adjust in the sound crossing timescale, either via evaporation or expansion (Schaye 2001; Lequeux 2005). The criterion assumes that the cloud is isolated, spherical, homogeneous, and exhibits no bulk motions.

The dynamical and sound crossing times, deduced cloud sizes, and cloud gas masses are listed in Table 4 for clouds $7+8,10$, and 11 , and the representative clouds 15,18 , and 20 for $\log n_{\mathrm{H}}=-3,-4$, and -5 . As described above, clouds $7+8$ are well constrained to have $\log n_{\mathrm{H}} \simeq-4$, whereas cloud 10 and the remaining clouds with only limits on the metal line column densities are not well constrained.

For $\log n_{\mathrm{H}} \leqslant-3,-4$, and -5 , we obtain $\tau_{\text {dyn }} \simeq 1 \times 10^{9}$, $3 \times 10^{9}$, and $1 \times 10^{10} \mathrm{yr}$, respectively. Note that $\tau_{\mathrm{dyn}} \propto n_{\mathrm{H}}{ }^{-1}$, whereas $\tau_{\mathrm{sc}} \propto\left(f_{\mathrm{H}_{\mathrm{I}}} n_{\mathrm{H}}\right)^{-1}$, and $\tau_{\text {cool }} \propto n_{\mathrm{H}}{ }^{-1 / 2}$. The relative behavior of these timescales is such that a transition from $\tau_{\mathrm{sc}}<\tau_{\text {dyn }}$ to $\tau_{\mathrm{sc}}>\tau_{\text {dyn }}$ occurs as $n_{\mathrm{H}}$ increases. This indicates that, for larger $n_{\mathrm{H}}$, the clouds have a propensity to be in the regime of Jeans instability, whereas as for smaller $n_{\mathrm{H}}$ the clouds become unstable to evaporation and expansion. The last column of Table 4 notes whether a cloud model is inferred to be Jeans unstable (JU) or unstable to expansion and/or evaporation (EE). The behavior with $n_{\mathrm{H}}$ suggests that between $-3 \leqslant \log n_{\mathrm{H}} \leqslant-5$ there is a density at which the clouds would classify as being in hydrodynamic equilibrium. This is also reflected in the behavior of the Jeans lengths, $L_{J}$, which would be equivalent to the absorption length scales, $L$, when $\tau_{\text {dyn }}=\tau_{\text {sc }}$.

Note that the absorption length scales, $L$, and the cloud gas masses, $M_{g}$, become unphysically large as hydrogen density decreases. This would suggest that the clouds have $\log n_{\mathrm{H}}>-5$, though we caution that the masses are probably overestimates due to the assumption of a spherical geometry. If more akin to cylindrical structures, viewed along the long axis, a factor of $\beta=0.1$ reduces the masses by two orders of magnitude. However, the absorption length scale along the line of sight is independent of geometry.

\footnotetext{
13 The Jeans mass, $M_{J}$, usually applies to the total mass. If the gas fraction, $f_{g}=\Omega_{b} / \Omega_{m}$, in these clouds is near the cosmic mean, then gas mass and total mass are related by $M_{\mathrm{tot}}=M_{g} / f_{g}$.
} 


\subsection{Hydrodynamic Equilibrium Conditions}

In view of the arguments given by Schaye (2001) that Ly $\alpha$ clouds should be in the regime of hydrostatic equilibrium, and in view of the unphysically large cloud length scales we deduce in the regime of Jeans instability, it is reasonable to explore the inferred cloud conditions under the assumption of hydrodynamic equilibrium. This assumption will yield an equilibrium value for $n_{\mathrm{H}}$, from which the ionization conditions and metallicities can be constrained.

Equating the dynamical time and the sound crossing time, and invoking the absorption length scale, $L=N(\mathrm{HI}) /\left(f_{\mathrm{H}_{\mathrm{I}}} n_{\mathrm{H}}\right)$, we derive the condition of hydrodynamic equilibrium,

$$
n_{\mathrm{H}} x_{\mathrm{H}} f_{g} \gamma k T\left[f_{\mathrm{HI}}\left(n_{\mathrm{H}}, T\right)\right]^{2}=G A_{\mathrm{H}} \mu m_{\mathrm{a}}^{2}[N(\mathrm{HI})]^{2} \text {, }
$$

where $N(\mathrm{HI})$ and $T$ are measured from the VP fit models to the Ly $\alpha$ profiles, and $f_{\mathrm{H}_{\mathrm{I}}}\left(n_{\mathrm{H}}, T\right)$ and $n_{\mathrm{H}}$ are computed using ionization models of the clouds.

Since the hydrogen ionization fraction is a function of $n_{\mathrm{H}}$, Equation (2) is a transcendental equation and must therefore be solved numerically for the equilibrium hydrogen density for a cloud with temperature $T$. For each cloud, we interpolate a grid of ionization models with metallicity $\log Z_{\mathrm{m}} / Z_{\odot}=-2$ for $-6.0 \leqslant \log n_{\mathrm{H}} \leqslant-2.0$ in steps of $\Delta \log n_{\mathrm{H}}=0.2$ and $2.6 \leqslant \log T \leqslant 6.0$ in steps of $\Delta \log T=0.1$. We set $T$ equal to the measured cloud temperature (based upon the Doppler $b$ parameter from the VP fits), interpolate to obtain $f_{\mathrm{HI}}\left(n_{\mathrm{H}}, T\right)$ as a function $n_{\mathrm{H}}$ at that $T$, and iterate using Brent's method to locate the $n_{\mathrm{H}}$ that satisfies Equation (2) to a tolerance $1 \times 10^{-10}$. The method assumes that $f_{\mathrm{HI}}\left(n_{\mathrm{H}}, T\right)$ is independent of metallicity (which we verified for $\log Z_{\mathrm{m}} / Z_{\odot} \leqslant-1$ ).

For this exercise, we treat clouds 7 and 8 separately (i.e., as a multi-phase structure as opposed to a single-phase cloud as done in Section 5.1). We also omit cloud 9, which has a very uncertain Doppler $b$ parameter in that its temperature is consistent with $T=0 \mathrm{~K}$.

Once Equation (2) is satisfied, the cloud absorption length scale, $L$, can be computed, from which the cloud gas mass can be estimated. Note that these quantities, while determined from the measured $N(\mathrm{HI})$ and $T$ as constrained by the ionization models, will be equivalent to the Jeans length and Jeans gas mass for the equilibrium $n_{\mathrm{H}}$.

For each cloud, the uncertainty in the equilibrium $n_{\mathrm{H}}$ and $f_{\mathrm{H}_{\mathrm{I}}}$ are obtained by accounting for the measured uncertainties in both $N(\mathrm{HI})$ and $T$ from the VP fits. The uncertainties in $L$ account for the uncertainties in $N(\mathrm{HI}), n_{\mathrm{H}}$, and $f_{\mathrm{H}_{\mathrm{I}}}$. The uncertainties in $M_{g}$ account for the uncertainties in $\rho_{g}$ (due to $\left.n_{\mathrm{H}}\right)$ and $L$.

Once the equilibrium values are determined, the cloud metallicities and their uncertainties can be estimated from the equilibrium ionization cloud model. Denoting this metallicity as $Z$ (in solar units), we have

$$
\log Z=\log \left[\frac{N(\mathrm{X})}{N(\mathrm{HI})}\right]_{\mathrm{VP}}+\log \left[\frac{Z_{\mathrm{m}} f_{\mathrm{HI}} n_{\mathrm{H}}}{n_{\mathrm{X}}}\right]_{\mathrm{eq}},
$$

where $N(\mathrm{HI})$ is the measured neutral hydrogen column density obtained from the VP fit to the data, $N(\mathrm{X})$ is the measured or upper limit on the column density species $\mathrm{X}$ from the VP modeling, and $n_{\mathrm{X}}$ is the number density of species $\mathrm{X}$ from the ionization model for the equilibrium $f_{\mathrm{HI}}$ and $n_{\mathrm{H}}$ and the model metallicity $Z_{\mathrm{m}}$. That is, we scale the equilibrium ionization model with $Z_{\mathrm{m}}$ to obtain $Z$ and an estimate of its uncertainty (which incorporates the "uncertainty" in $N(\mathrm{X})$ for the upper limits).

In Figure 8, we present the deduced cloud properties under the assumption of hydrodynamic and ionization equilibrium. Solid data points satisfy the condition of hydrogen ionization equilibrium, whereas open data points do not. The measured $N(\mathrm{HI})$ and $T$ are shown in panels (a) and (b), respectively. The equilibrium $n_{\mathrm{H}}$ and $f_{\mathrm{H}_{\mathrm{I}}}$ are presented in panels (c) and (d), respectively. Note that, as suggested by the analysis presented in Table 4 , the clouds have $-4.8 \leqslant \log n_{\mathrm{H}} \leqslant-3.8$ and $-6 \leqslant \log f_{\mathrm{HI}} \leqslant-4$.

The metallicities are plotted in Figure 8(e). Except for clouds 7,8 , and 10 , the metallicities are upper limits. For the assumption of single ionization phase clouds, each metal line provides a unique limit. The most stringent limits are presented, with the data point color coded by the ion that provides this best limit (red for $\mathrm{O}^{+5}$, green for $\mathrm{C}^{+3}$, and blue for $\mathrm{C}^{+2}$ ). In general, the upper limits are $\log Z / Z_{\odot}<-1.7$. The measured values for clouds 7,8 , and 10 are $\log Z / Z_{\odot}=-1.65_{-0.10}^{+0.14},-2.44_{-0.57}^{+0.79}$, and $-1.78_{-0.03}^{+0.20}$, respectively.

The absorption length scale (which is equal to the Jeans length under the assumption of hydrodynamic equilibrium), is shown in Figure $8(\mathrm{~h})$ for each cloud. The typical length scale is few hundred kpc, except for clouds 2, 3, 4, and 5, which have the lowest temperatures and thus relatively high densities and low ionization conditions. Recall that the VP fits for clouds $1-6$ should be viewed with caution. The gas masses, $M_{g}$, of the clouds (which equal the Jeans gas masses under the assumption of hydrodynamic equilibrium) are presented in Figure $8(\mathrm{~g})$. Assuming spherical clouds, the gas masses are on the order of $10^{8}-10^{10} M_{\odot}$, except for cloud 11 , which has $M_{g} \simeq 10^{11} M_{\odot}$. These mass estimates should be viewed as upper limits by as much as one to two orders of magnitude. Cloud 11 is the highest temperature cloud with the highest ionization condition. The larger mass is a result of the large length scale and the fact that the hydrogen density is relatively high, $\log n_{\mathrm{H}} \simeq-4.1$.

The cooling time, $\tau_{\text {cool }}$, is presented in Figure $8(\mathrm{~h})$. Note that under the assumption of hydrodynamic equilibrium, the clouds have thermal stability in times the order of 1-2 orders of magnitude shorter than the Hubble time. The collisional time, $\tau_{\text {coll }}$, is presented in panel (i). Except for clouds 10 and 14, the clouds are in ionization equilibrium. However, clouds 2-6 also may not be in ionization equilibrium; we again remind the reader that the VP fits to these latter clouds are to be viewed with caution. The dynamical time (which is equal to the sound crossing time under the assumption of hydrodynamic equilibrium), is presented in Figure 8(j) for each cloud. In all cases, $\tau_{\text {dyn }}$ and $\tau_{\mathrm{sc}}$ are a factor of a few less than the Hubble time, which is indicated by the dashed line.

The values obtained from this exercise are in remarkable agreement with those predicted from the simple scaling relations proposed by Schaye (2001) for the assumption of hydrodynamic equilibrium.

For comparison between the individual models of clouds 7 and 8 and the combined clouds 7+8 modeled in Section 5.1, we indicate the results of the latter analysis as dashed boxes on Figure 8 . For clouds $7+8$, the measured column densities were summed, from which the density and metallicity were simultaneously constrained assuming a single ionization phase (see Figure 6). That analysis resulted in a slightly larger $n_{\mathrm{H}}$ than the assumption of hydrodynamic equilibrium in the individual clouds, through the metallicities are consistent between analysis 

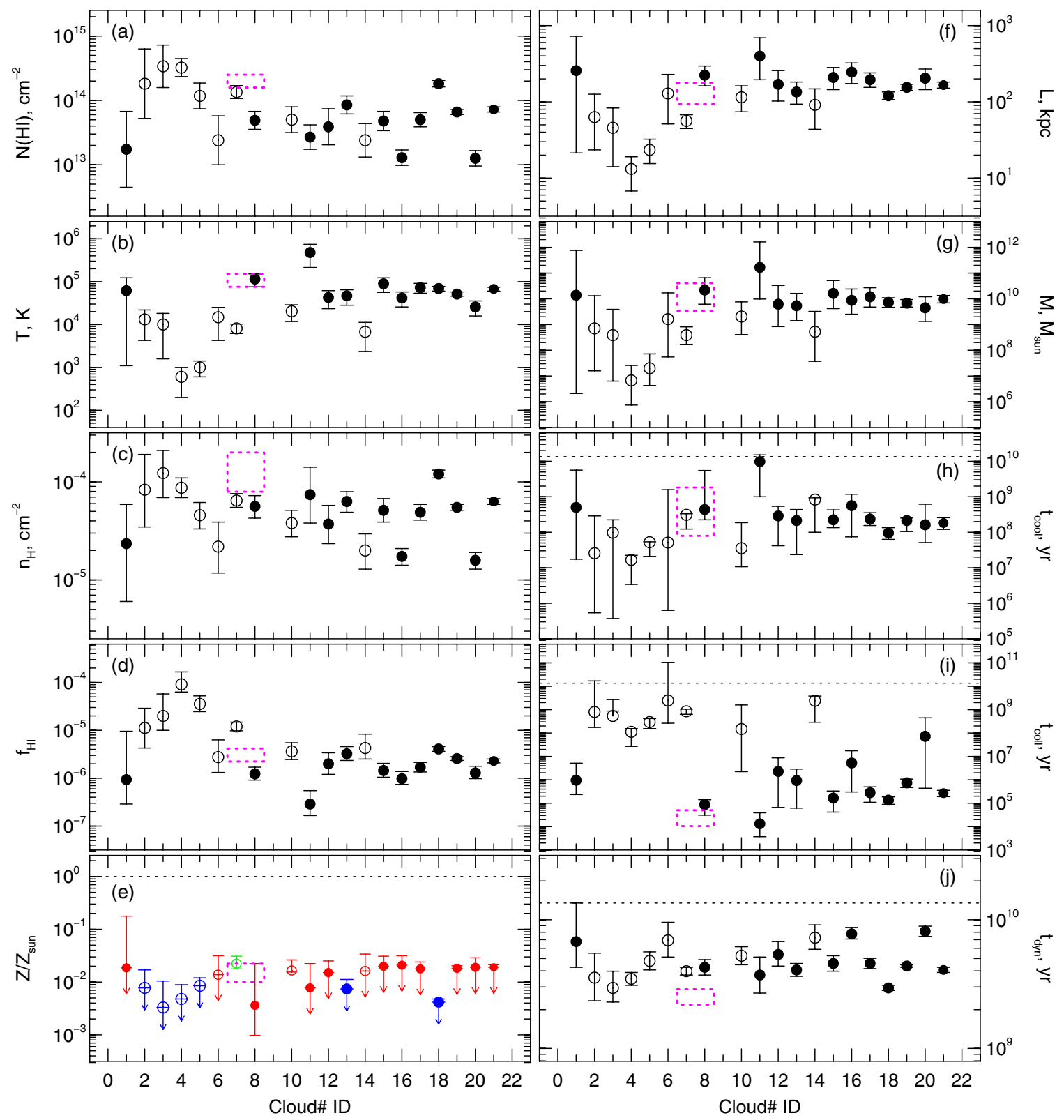

Figure 8. Deduced cloud physical conditions under the assumption of hydrodynamic equilibrium. (a) The measured $N\left(\mathrm{HI}_{\mathrm{I}}\right)$ from the VP fits. (b) The measured temperature, based upon the VP Doppler $b$ parameter. (c) The equilibrium $n_{\mathrm{H}}$ computed from Equation (2). (d) The equilibrium hydrogen ionization fraction, $f_{\mathrm{H}}$, computed from Equation (2). (e) The metallicity, $Z / Z_{\odot}$, computed from Equation (3): the points are colored coded based upon the ion that provides the most stringent constraints, red for $\mathrm{O}^{+5}$, green for $\mathrm{C}^{+3}$, and blue for $\mathrm{C}^{+2}$. The dotted line is solar. (f) The absorption length scale, $L=N\left(\mathrm{H}_{\mathrm{I}}\right) /\left(f_{\mathrm{H}_{\mathrm{I}}} n_{\mathrm{H}}\right)$. Note that this is equivalent to the Jeans length, $L_{J}$, for the assumption of hydrodynamic equilibrium. (g) The gas mass, $M_{g}$. Note that $M_{g}=M_{J}$, the Jeans gas mass, for the assumption of hydrodynamic equilibrium. (h) The cooling time, $\tau_{\text {cool }}$; the dotted line is the Hubble time, $\tau_{\mathrm{H}}=1 / H_{0}$. (i) The collisional time, $\tau_{\text {coll }}$; the dotted line is $\tau_{\mathrm{H}}$. (j) The dynamical time, $t_{\mathrm{dyn}}$; the dotted line is $\tau_{\mathrm{H}}$. Note that the sound crossing time, $\tau_{\mathrm{sc}}$ is equivalent to $\tau_{\mathrm{dyn}}$ for the assumption of hydrodynamic equilibrium. Open data points represent clouds that do not satisfy the criterion of hydrogen ionization equilibrium, whereas solid data points do satisfy the criterion. Dotted magenta boxes straddling clouds 7 and 8 represent the single-phase model presented in Section 5.1 as illustrated in Figure 6.

(A color version of this figure is available in the online journal.)

methods. The difference in $n_{\mathrm{H}}$ is likely due to the adding of the column densities. We also compare the individual cloud equilibrium and the combined cloud $L, M_{g}, \tau_{\text {cool }}$, and $\tau_{\text {dyn }}$ $\tau_{\text {coll }}$, shown as the dashed boxes on Figures 8(f)-(j). Note that cloud 7 has $\tau_{\text {coll }} \gg \tau_{\mathrm{ph}}$, where $\tau_{\mathrm{ph}}=10^{4} \mathrm{yr}$, indicating that the hydrogen in this cooler cloud is photoionized. On the other hand, cloud 8 has $\tau_{\text {coll }} \simeq \tau_{\text {ph }}$ for both hydrogen and $\mathrm{O}^{+5}$, and thus has a substantial collisional ionization contribution.

Cloud 11 is of particular interest. This component is the hottest and most highly ionized, $f_{\mathrm{HI}} \simeq 3 \times 10^{-7}$, cloud in the complex, and is among the highest density, $\log n_{\mathrm{H}} \simeq-4.1$, of the clouds. We find $\tau_{\text {coll }} / \tau_{\mathrm{ph}} \simeq 1$ for hydrogen, and $\tau_{\text {coll }} / \tau_{\mathrm{ph}}<$ 100 for $\mathrm{O}^{+5}$; thus, hydrogen is equally photo- and collisionally ionized, whereas $\mathrm{O}^{+5}$, though not detected, is predominantly collisionally ionized. It is plausible that this cloud (cloud 11) is shock-heated gas, as further suggested by the fact that the deduced cooling time $\left(\tau_{\text {cool }} \simeq 10 \mathrm{Gyr}\right)$ is substantially longer than the deduced dynamical time $\left(\tau_{\text {dyn }} \simeq 4 \mathrm{Gyr}\right)$. Crudely adopting the dynamical time as a proxy for the compression time (e.g., Birnboim \& Dekel 2003; Dekel \& Birnboim 2006), and considering the temperature and ionization conditions, we 
find that cloud 11 is the only component in the H I complex that is suggestive of shocked gas.

\subsection{Caveats}

The analysis we have presented has employed many simplifying assumptions. The VP fitting method philosophy assumes that the gas structure comprises several spatially distinct isothermal clouds. In fact, it is very possible that the H I complex is a quasi-continuous non-uniform structure with temperature and density variations having a range of bulk motions (and possibly at least one shock front, i.e., cloud 11). It is also plausible that such bulk motions can align in line-of-sight velocities creating caustics that emulate distinct clouds so that some VP components actually model a heterogeneous physical condition.

Furthermore, the analysis invoking the dynamical time, sound crossing time, and Jeans mass and length is predicated on a homogeneous cloud. A cloud in thermodynamic equilibrium cannot simultaneously be homogeneous and isothermal, as we have assumed here.

In support of the assumption of hydrodynamic equilibrium, we note that Schaye (2001) argues that a spherical cloud with an isothermal density profile, i.e., $n_{\mathrm{H}}(r) \propto r^{-2}$, has a welldefined characteristic $n_{\mathrm{H}}$ that is on the order of the maximum density probed by the line of sight. For the gas mass estimates, we have assumed spherical clouds, which is probably a very poor assumption. As such, the estimated gas masses should be considered upper limits.

Finally, the ionization modeling assumes photoionization and collisional ionization equilibrium, which we have shown to be a valid condition for the majority, but not all, of the clouds. In addition, the metallicity estimates are based upon the assumption of single-phase ionization conditions. If some of the plausible concerns expressed above with regard to heterogeneous physical conditions aligned in line-of-sight velocity hold, then multi-phase structure could be present that would affect the metallicity estimates. Overall, the assumption of ionization equilibrium in single-phase gas is critical to all deduced quantities, especially the metallicities and the thermal equilibrium values presented in Section 6.5.

\section{DISCUSSION}

With a velocity extent of $1600 \mathrm{~km} \mathrm{~s}^{-1}$, the $\mathrm{HI}_{\mathrm{I}}$ absorption complex at $z=0.672$ in the quasar Q1317+277 is a most intriguing gaseous structure. Absorption with velocity spreads on the order of $2000 \mathrm{~km} \mathrm{~s}^{-1}$ occur in approximately $10 \%-15 \%$ of quasars (Weymann et al. 1991; Gibson et al. 2009). In almost all cases, extreme absorption of this nature is produced by material ejected from the quasar itself (i.e., broad absorption line quasars); however, the properties of the $z=0.672 \mathrm{H}$ i complex studied here are not suggestive of absorption "associated" with or "intrinsic" to the quasar. For example, the Hi exhibits no evidence of partial covering, which is an adopted signature of associated gas (Barlow \& Sargent 1997; Ganguly et al. 1999). Furthermore, the kinematics of the metals' absorption lines are kinematically similar to the velocity spreads observed in galaxy halos (Churchill \& Vogt 2001; Churchill et al. 2003). Thus, the H I complex is likely intervening absorption. Early on, Bahcall \& Salpeter (1965) suggested that the environments of galaxy clusters may give rise to extensive, intervening broad absorption line complexes, but few potential candidates have been identified.
In this section, we summarize and further examine the nature and environment of the $z=0.672 \mathrm{H}$ I complex toward Q1317+277, compare it to other similar Hi complexes, and discuss the possible origin of the Q1317+277 H I complex, such as hot-mode or cold-mode accretion, galactic winds, accreting filaments, intracluster gas, and/or the warm-hot phase of the ionized medium (WHIM).

\subsection{The Nature of the $z=0.672$ H I Complex}

The Q1317+277 H I complex at $z=0.672$ is characterized by a velocity spread of $1600 \mathrm{~km} \mathrm{~s}^{-1}$ and $21 \mathrm{Ly} \alpha$ components with $12.9 \leqslant \log N(\mathrm{HI}) \leqslant 14.5$, as determined by VP fitting. Under the assumption that the components (clouds) are near hydrodynamic equilibrium, the temperatures, hydrogen number densities, and hydrogen ionization fractions primarily range between $10^{4} \mathrm{~K} \leqslant T \leqslant 10^{5} \mathrm{~K},-3.9 \leqslant \log n_{\mathrm{H}} \leqslant-4.9$, and $-5.5 \leqslant \log f_{\mathrm{H}_{\mathrm{I}}} \leqslant-6.0$. The deduced cloud sizes are on the order of $200 \mathrm{kpc}$, and the cloud baryonic gas masses range between $10^{9}-10^{10} M_{\odot}$. Because the gas masses scale as $n_{\mathrm{H}}^{-2} f_{\mathrm{H}}^{-3}$, the large masses result from the low hydrogen number densities and the high ionization conditions of the clouds.

The metallicities are measured only for clouds 7,8 , and 10 and are $\log Z / Z_{\odot}=-1.7,-2.5$, and -1.8 , respectively. Upper limits on the remaining clouds are $\log Z / Z_{\odot}<-2.4$ to $\log Z / Z_{\odot}<-1.7$. The limits on the cloud metallicities do not rule out enrichment at the level of the high-redshift IGM (e.g., Cowie \& Songaila 1998; Simcoe et al. 2004). On the other hand, the low metallicities are 1-2 orders of magnitude below the $\log Z / Z_{\odot} \sim-0.6$ metallicities measured in $z=0.7 \mathrm{X}$-ray clusters (Balestra et al. 2007; Maughan et al. 2008).

Further insight is gained by examination of the kinematicionization substructure. Near the velocity center of the complex, at $v \simeq 75 \mathrm{~km} \mathrm{~s}^{-1}$ with respect to the galaxy $\mathrm{G} 1$, is a hot $T=480,000 \mathrm{~K}$, collisionally ionized component (cloud 11), which is very likely shocked gas. The narrow velocity region just blueward of cloud 11 comprises four clouds, within which the only metal lines are detected. The general overall absorption morphology of these four components (clouds 7-10) is that of a double profile suggesting two absorbing structures contiguous in velocity space. Cloud 10 , separated by $\simeq 50 \mathrm{~km} \mathrm{~s}^{-1}$ from cloud 11 , has detected O VI absorption, which is deduced to arise in cool $T=20-30 \mathrm{~K}$ photoionized gas. Clouds 7 and 8, which have detected $\mathrm{O}$ VI, $\mathrm{C}$ IV, and $\mathrm{C}$ III absorption, give rise to a single profile, which is best modeled with a narrow core (cloud 7, cool photoionized gas with $T \simeq 10,000 \mathrm{~K}$ ) and a broad component (cloud 8, hot collisionally ionized gas with $T=115,000 \mathrm{~K}$ ). The velocity centroids are separated by less than half of a single COS spectral resolution element of $\simeq 17 \mathrm{~km} \mathrm{~s}^{-1}$. In addition, cloud 9 appears to be a very narrow $(T<10,000 \mathrm{~K})$ blue wing of cloud 10 offset by $\simeq 20 \mathrm{~km} \mathrm{~s}^{-1}$ that overlaps with the red wing of cloud 8 . These substructures may be suggestive of clouds moving through a hot, $T>10^{6} \mathrm{~K}$, medium in which a conductive interfaces arises at the boundary between the cool, warm, and hot gas (e.g., Sembach et al. 2003).

Knowing the environment of the $\mathrm{H}$ I complex and relationship to galaxies would be instrumental for a broader interpretation. The proximate galaxy is $\mathrm{G} 1$, which lies at $D=58 \mathrm{kpc}$ from the quasar line of sight and has a redshift very near the mean of the H I complex. The virial mass of galaxy G1 is estimated to be within a factor of two of the virial mass of M87 $\left(M_{\text {vir }} / M_{\odot} \simeq 10^{14}\right.$, Strader et al. 2011), suggesting that galaxy G1 could be a central galaxy in a Virgo-like cluster. However, 
we find no clearly compelling evidence that $\mathrm{G} 1$ resides in a galaxy cluster or in a group with an X-ray-emitting intracluster medium.

A search of the NASA Extragalactic Database and SIMBAD database yielded no reported X-ray measurements of Q1317+277. Within 4' of Q1317+277, there are no sources in the ROSAT all-sky survey bright source catalog (Mickaelian et al. 2006; Voges et al. 1999). Of the five closest X-ray sources that are not identified either as a star or an active galactic nucleus/quasar (which have known redshifts), one (1RXS $\mathrm{J} 131954.5+253210)$ lies at $2^{\circ}$ from Q1317+277 (50 Mpc projected at $z=0.672$ ). The bright $R=15.1$ galaxy identified within $29^{\prime \prime}$ of the X-rays would be $2 \mathrm{Mpc}$ projected from this source at $z=0.672$. If the $\mathrm{X}$-ray source is associated with this galaxy, it is likely that the galaxy and X-ray source reside at a redshift much lower than the H I complex.

In the ROSAT HR1 band, a minimum of 0.04 counts $\mathrm{s}^{-1}$ is required for a source to be included in the ROSAT catalog. Applying this upper limit, and invoking the relationship (Mullis 2001) between the count rate and the total flux in the band (accounting for the aperture correction), we estimate $L_{\mathrm{X}}<$ $2 \times 10^{39} \mathrm{erg} \mathrm{s}^{-1}$. This is four orders of magnitude below the expected X-ray luminosity of $2 \times 10^{43} \mathrm{erg} \mathrm{s}^{-1}$ for a cluster with a central galaxy of virial mass of G1, where we have employed the bolometric X-ray luminosity to virial mass scaling relation of Bryan \& Norman (1998) and corrected for the X-ray band (see Mullis 2001). The virial temperature of galaxy G1 is estimated to be on the order of $10^{7} \mathrm{~K}$, which yields a coronal temperature of $k T \simeq 0.8 \mathrm{keV}$. According to the compilation of Crain et al. (2011), our upper limit on $L_{X}$ is not inconsistent with the observed X-ray luminosities of early-type galaxies with similar $k T$.

We have successfully measured spectroscopic redshifts for only galaxies G1 and G2. As such, we cannot directly deduce the presence of a cluster at $z=0.672$, nor estimate the velocity dispersion of the galaxies that may reside at this redshift. Based upon the photometric properties examined in the imaging data, it is difficult to definitively rule out or favor the presence of a cluster at $z=0.67$. However, the upper limits on the X-ray flux within $50 \mathrm{Mpc}$ projected from Q1317+277 and the low metallicity of the H I complex, 1-2 dex below intracluster gas measurements (Balestra et al. 2007; Maughan et al. 2008), do not favor a large cluster nor a hot intracluster medium.

Such considerations leave open the possibility that the $\mathrm{HI}$ complex may be a phenomenon closely linked to a massive old elliptical galaxy that is not in an overdense environment. Taken together at face value, the data and the results of our analysis suggest a low-metallicity structure, possibly a filament or the remnants of a disrupted filament. There remains the question of the possible connection to the smaller galaxy G3, such as, for example, a scenario in which a superbubble gives rise to the absorption. However, it would not be expected that a superbubble structure would extend over $1600 \mathrm{~km} \mathrm{~s}^{-1}$. On the other hand, if the metal lines were due to a superbubble structure, higher metallicities would be expected. Unfortunately, galaxy G3 does not have a measured or estimated redshift and so we cannot further speculate on the possible contribution from galaxy G3.

\subsection{Review of Comparable H I Complexes}

Given the dramatic velocity spread and kinematics of the H I complex toward Q1317+277, and given its proximity to galaxy G1 (and possibly G3), it is of interest to investigate how rare/common are such absorbing complexes, what their observed relationships are with respect to galaxies, and what interpretations have been adopted in view of the role of gas in the evolution of individual and group galaxies. Such insights may help identify the Q1317+277 H I complex in a broader context.

One example is the $z \simeq 2 \mathrm{C}$ IV absorber complex toward the "Tololo Pair" (Tol 1037-27, $z_{\mathrm{em}}=2.18$, and Tol 1038-27, $z_{\mathrm{em}}=2.33$ ), which may be produced by intracluster gas (Jakobsen et al. 1986). The C IV doublets, later observed in two additional quasars in proximity on the sky, exhibit multiple discrete components with velocity widths ranging between 50-1000 $\mathrm{km} \mathrm{s}^{-1}$ and may extend some $18 \mathrm{Mpc}$ (Dinshaw \& Impey 1996).

Another possible intracluster absorption complex, at $z=$ 0.695 toward the $z_{\mathrm{em}}=1.05$ quasar PG 2302+029 (Jannuzi et al. 1996), exhibits broad ( $\left.\Delta v=3000 \mathrm{~km} \mathrm{~s}^{-1}\right)$ high ionization $\mathrm{C}$ IV, N v, and O vi doublets. In the FOS spectrum (velocity resolution $\Delta v=230 \mathrm{~km} \mathrm{~s}^{-1}$ ), the Ly $\alpha$ absorption is segregated into multiple individual components each with $\Delta v<250 \mathrm{~km} \mathrm{~s}^{-1}$ distributed across the full velocity range of the metals. Near the central velocity, narrow $\mathrm{C}$ IV, $\mathrm{Nv}$, and $\mathrm{O}$ vi are present in one $\operatorname{Ly} \alpha$ component. No low ionization species are present. Jannuzi et al. (1996) suggest three possible interpretations: (1) material ejected from the quasar at extreme ejection velocity, (2) material associated with galaxies or the intracluster medium of a cluster or supercluster of galaxies, and (3) remnant material from supernovae in a galaxy. Unfortunately, their observations did not provide data capable of distinguishing between these scenarios.

Toward the $z_{\mathrm{em}}=0.297$ quasar H1821+643, Tripp et al. (2001) reported an $\mathrm{HI}$ complex at $z=0.1212$ comprising five Ly $\alpha$ components distributed over a velocity interval of $\sim 700 \mathrm{~km} \mathrm{~s}^{-1}$ in high-resolution STIS and FUSE spectra. The $\log N(\mathrm{HI})$ column densities range from 12.7-13.8. Absorption from $\mathrm{OVI}$ is present in a single broad wing of the central component, for which collisional ionization is favored with $T=10^{5.3}-10^{5.6} \mathrm{~K}$ and $-1.8 \leqslant[\mathrm{O} / \mathrm{H}] \leqslant-0.6$. Seven galaxies in the velocity range of the absorption are present at impact parameters ranging from $140-2400 \mathrm{kpc}$, with the $140 \mathrm{kpc}$ galaxy aligned in redshift with the O vi absorption. Tripp et al. (2001) favor the scenario in which the $\mathrm{H}$ I complex is intragroup gas or an unvirialized filamentary structure.

Using GHRS, STIS, and FUSE spectra of the $z_{\mathrm{em}}=0.116 \mathrm{BL}$ Lac object PKS 2155-304, Shull et al. (1998, 2003) analyzed an $\mathrm{H}$ I complex with $14 \mathrm{Ly} \alpha$ components centered at $z=0.056$ spread over a velocity interval of $2270 \mathrm{~km} \mathrm{~s}^{-1}$. They estimate the $\mathrm{H}$ I column densities have the range $14.5 \leqslant \log N(\mathrm{HI}) \leqslant 15.0$ with $\log Z / Z_{\odot}<-2.5$ and cloud depths less than $400 \mathrm{kpc}$. Five $\mathrm{H}$ I-emitting galaxies are found in the range $0.056 \leqslant z \leqslant 0.058$ with impact parameters $400-790 \mathrm{kpc}$. The two strongest Ly $\alpha$ blends have detectable $\mathrm{O}$ VI and possible O VIII absorption measured in a Chandra spectrum. If the gas is the warm-hot ionized medium (WHIM) then the density is constrained to $\log n_{\mathrm{H}} \simeq-4$. Shull et al. (2003) favor a scenario in which the O VI arises in "nearside" and "backside" shocked infall into the potential well of the galaxy group.

In high-resolution STIS and FUSE spectra of the $z_{\mathrm{em}}=0.370$ quasar HS 0624+6907, Aracil et al. (2006a) report a cluster of $13 \mathrm{Ly} \alpha \operatorname{lines}^{14}$ at $z=0.0635$ with a velocity spread

\footnotetext{
14 An "erratum" to Aracil et al. (2006a) was published (see Aracil et al. 2006b). However, the deduced properties of the H I absorbing complex are unaltered.
} 
of $1000 \mathrm{~km} \mathrm{~s}^{-1}$. The $\mathrm{HI}$ column densities range between $12.6 \leqslant \log N(\mathrm{HI}) \leqslant 15.3$. Only in the central component, with total $\log N(\mathrm{HI})=15.4$, are metal lines detected (Si III, Si IV, and C IV, but no O VI) from which the gas is deduced to be photoionized with metallicity $\log Z / Z_{\odot}=-0.05$, very near to solar enrichment, with $\log n_{\mathrm{H}}=-3.9$. The gas temperatures are deduced to be $T<10^{5} \mathrm{~K}$. The estimated baryonic mass of this component is $\sim 10^{5} M_{\odot}$ with an absorption length scale of $3-5 \mathrm{kpc}$. They report 10 galaxies within $135-1370 \mathrm{kpc}$ in the range $0.062 \leqslant z \leqslant 0.067$, but this group is not consistent with elliptical-rich groups. On account of the high metallicity and cool temperatures, Aracil et al. (2006a) favor the interpretation that this H complex is tidally stripped material from one of the nearby galaxies.

The H I complexes toward H1821+643, PKS 2155-304, and HS 0624+6907 have both similarities and differences with the H I complex toward Q1317+277. However, the Q1317+277 H I complex bears little resemblance to the metal-rich complexes observed toward PG 2303+029 and toward the Tol 1037-27 and Tol 1038-27 pair. These latter two complexes may be examples of metal-enriched intracluster gas.

The broad H I component in the complex toward H1821+643 exhibits O VI that is likely to be predominantly collisionally ionized with a relatively high metallicity. Similarly, cloud 8 in the Q1317+277 H I complex appears to be a $T>10^{5} \mathrm{~K}$, collisionally ionized $\mathrm{O}$ VI absorber, but accompanied by $\mathrm{C}$ IV and $\mathrm{C}$ III absorption. On the other hand, the hottest, broad component in the Q1317+277 H I complex has no detected O VI and has upper limits on metallicity indicating that it is metal poor in comparison. The H1821+643 O VI absorber is at a substantially larger impact parameter to the nearest galaxy, which resides in a group that clearly has no massive elliptical galaxy, whereas the Q1317+277 H I complex is very close in projection to the massive elliptical galaxy G1.

The $\mathrm{H}$ I column density for the low-metallicity $\mathrm{O}$ VI absorber in the complex absorption toward PKS 2155-304 is 1-2 orders of magnitude greater than the $N(\mathrm{HI})$ of the components in the Q1317+277 H I complex. However, the clouds have similar $n_{\mathrm{H}}$. In both complexes, the $\mathrm{O}$ VI resides in the wings of the largest Ly $\alpha$ components. Shull et al. (2003) interpret this as a shock interface, and this interpretation may apply in the case of the Q1317+277 H I complex. However, as with the H1821+643 O vi absorber, the environment of the PKS 2155-304 H I complex resides within a moderate group of galaxies having no massive elliptical galaxy.

Of the three examples, the H I complex toward HS 0624+6907 has an $\mathrm{H}$ I absorption profile morphology most similar to that of the Q1317+277 Hi complex. The cool photoionized cloud with CIV, Si IV, and Si III absorption compares to the cool photoionized O VI absorbing cloud 10 in the Q1317+277 H I complex, but cloud 10 has no Si IV or C IV absorption. Though the clouds have similar $n_{\mathrm{H}}$, cloud 10 has lower $N(\mathrm{HI})$, higher ionization conditions, and a much lower metallicity as compared to the nearly solar metallicity cloud in the HS 0624+6907 complex. Because of the low ionization deduced for the latter cloud, the mass is $\sim 4$ orders of magnitude smaller than the mass deduced for cloud 10. Again, the galaxy environment of the HS 0624+6907 H I complex contains no massive elliptical galaxy.

Overall, two unique features to the Q1317+277 H I complex are that it is at substantially higher redshift $(z=0.672)$ compared to the other reported $\mathrm{H}$ i complexes $(z=0.056,0.064$ and 0.121 ) and that it is in close projected proximity to a region dominated by a massive, metal-rich elliptical with an old stellar population.

\subsection{Interpreting the $z=0.672 \mathrm{H}_{\text {I Complex }}$}

Summarizing the gas properties of the Q1317+277 H I complex, we find (1) a hot, photo- and collisionally ionized component that is consistent with shocked gas, (2) a cool component with photoionized O VI absorption, and (3) a cool component plausibly layered within a warm component that is both photo- and collisionally ionized and exhibits O VI, C IV, and C III absorption, (4) several additional warm H I components spread over $1600 \mathrm{~km} \mathrm{~s}^{-1}$ in the rest frame of $z=0.672$, and (5) measurements of and limits on metal enrichment between $-2.5 \leqslant \log Z / Z_{\odot} \leqslant-1.7$.

If the rest-frame velocity spread of the Hi complex is due to the local Hubble flow, then the line-of-sight proper length of the structure would be $D_{\mathrm{HF}}=\Delta v / H_{0} E(z)$, where $\Delta v=1600 \mathrm{~km} \mathrm{~s}^{-1}$ and $E^{2}(z)=\Omega_{m}(1+z)^{3}+\Omega_{\Lambda}$. At $z=0.67$, we estimate $D_{\mathrm{HF}} \sim 15 \mathrm{Mpc}$. Based upon the ionization modeling, the deduced physical sizes of the H I complex are not consistent with a single structure of this extent. If the velocities of the $\mathrm{H}$ I absorbers are due to Hubble flow, then the complex must comprise absorbers that are spatially segregated; there are five main absorption features apparent in the Ly $\alpha$ profile, which would imply an average separation of $3 \mathrm{Mpc}$ and that we have by chanced probed several isolated absorption systems. Considering the similarities of the properties of the H I complexes toward H1821+643, PKS 2155-304, and HS $0624+6907$, and that they are clearly connected with galaxies on scales of $0.1-1 \mathrm{Mpc}$, we do not favor the interpretation that the Q1317+277 H I complex is multiple individual absorbers tracing a $15 \mathrm{Mpc}$ Hubble flow.

Is it reasonable that the individual peaks in the $\mathrm{H}_{\mathrm{I}}$ column density are a result of having fragmented from a single structure? If this structure were Jeans unstable, i.e., $\tau_{\text {dyn }} \ll \tau_{\text {sc }}$, then fragmentation and/or shock disruption into the observed components would be plausible.

Crudely modeling this hypothetical single structure as having the $N(\mathrm{HI})$ weighted mean temperature of the components, $\langle\log T\rangle=4.65$ (prior to fragmentation or shock disruption, where the latter could subsequently heat the shocked components), and assuming that the length of its long axis is the sum of the Jeans lengths for each of the components, we find that $\tau_{\text {dyn }} \simeq 1 / 20 \tau_{\mathrm{sc}}$ independent of the assumed mean hydrogen density, $\bar{n}_{\mathrm{H}}$, where $\tau_{\text {dyn }} \simeq 3 \times 10^{9}\left(\bar{n}_{\mathrm{H}} / 10^{-4}\right)^{1 / 2}$ and $\tau_{\mathrm{sc}} \simeq 6 \times 10^{10}\left(\bar{n}_{\mathrm{H}} / 10^{-4}\right)^{1 / 2} \mathrm{yr}$. The model is consistent with the plausibility that the multiple component structure of the H I absorption complex could very well have resulted from the fragmentation of a quasi-coherent single gaseous structure.

Our simple model yields a total Jeans length of $L_{J} \simeq$ $2\left(\bar{n}_{\mathrm{H}} / 10^{-4}\right)^{1 / 2} \mathrm{Mpc}$ and a total Jeans mass of $M_{J} \simeq 2 \times$ $10^{11}\left(\bar{n}_{\mathrm{H}} / 10^{-4}\right)^{1 / 2} \mathrm{Mpc}$, where the total mass is simply the sum of the Jeans masses for the individual components. The implied aspect ratio $\beta=2 R / L$ for a cylindrical structure is $\beta \simeq\left(M_{J} / \bar{\rho}_{g} L_{J}^{3}\right) \simeq 0.1$. If $\log \bar{n}_{\mathrm{H}}=-4$, then the H i complex could be crudely envisioned as a $10^{11} M_{\odot}$ mass cylindrical filament $2 \mathrm{Mpc}$ long with a $200 \mathrm{kpc}$ diameter. These values would scale as $\left(\bar{n}_{\mathrm{H}}\right)^{1 / 2}$.

Summarizing the luminous environment of the H I complex, we find (1) a massive, red, high-metallicity, elliptical galaxy (G1) with an old stellar population $(\sim 6 \mathrm{Gyr})$ residing at $D=58 \mathrm{kpc}$ and aligned in velocity between the shocked $\mathrm{H}_{\mathrm{I}}$ 


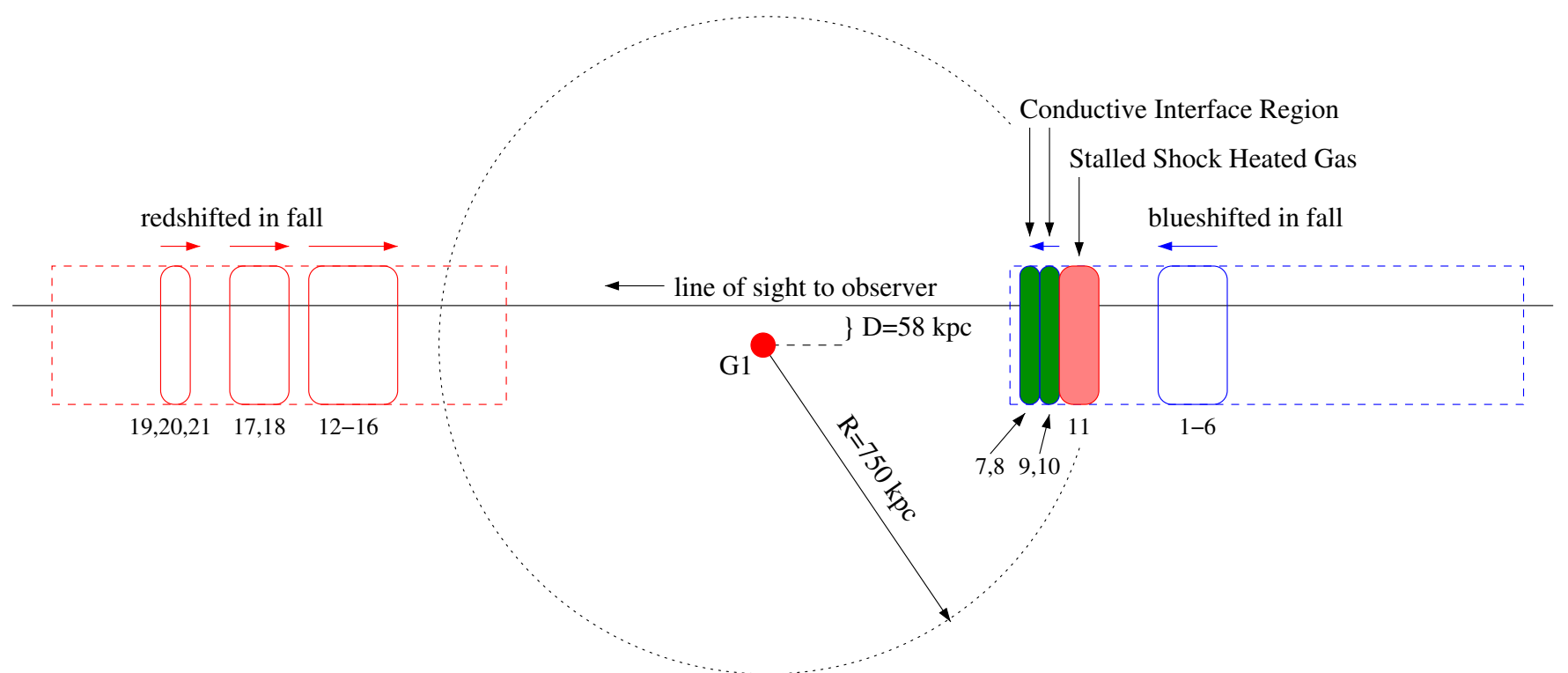

Figure 9. Schematic model illustrating the interpretation of the $\mathrm{H}$ I absorbing complex as an accreting filament onto the galaxy G1. The positive velocity material would require that it is infalling from the observer's side of the galaxy, whereas the negative velocity material would be infalling opposite the observer's side of the galaxy. The plausible relative physical locations of the absorbing clouds, labeled by their VP component numbers (see Table 2), are shown. The conductive interface (clouds 7-10) continues its infall after being stalled in a shock front, which is presently coincident with cloud 11 (see the text). The model is adapted from simulation results (e.g., see the "cold only" panel of Figure 6 in Kereš et al. 2009).

(A color version of this figure is available in the online journal.)

component and the metal-enriched $\mathrm{H}$ i components, near the $\mathrm{H}$ I profile velocity centroid, and (2) the upper limits on the X-ray luminosity consistent with the measured range for ellipticals with $k T \simeq 0.8 \mathrm{keV}$ and well below expected values for clusters dominated by massive ellipticals.

In addition to the X-ray data not supporting the idea that galaxy G1 resides in a large cluster, the metallicity of the $\mathrm{H} \mathrm{I} \mathrm{ab-}$ sorbing gas is constrained to be 1-2 orders of magnitude below the average enrichment of clusters at $z=0.7$ (Balestra et al. 2007; Maughan et al. 2008). The observations of Brinchmann \& Ellis (2000) and van der Wel et al. (2005) indicate that old, massive, metal-rich late-type galaxies were present in the field as early as $z=2-3$. The stellar population models for galaxy G1 suggest a formation epoch at $z \simeq 4$. It may be that galaxy G1 is not a dominant elliptical galaxy of a large group, but may be a galaxy in the field that formed at high redshift.

In addition to the aforementioned supporting observations, a massive, red, metal-rich elliptical far from a cluster environment is theoretically plausible. The cosmological simulations of Gabor \& Davé (2012), which treat accretion and feedback processes, yield a substantial fraction of red, high-mass galaxies independent of overdensity. Johansson et al. (2012) demonstrate that massive early-type galaxies are built in two phases. The first is an initial growth via in situ star formation fed by cold accretion $(z>3)$ that is later quenched via virial shocking (e.g., Birnboim \& Dekel 2003; Kereš et al. 2005, 2009; Dekel \& Birnboim 2006; van de Voort et al. 2011; van de Voort \& Schaye 2012). The second phase is accretion of stellar material $(z \sim 1.5-0.5)$, called "dry minor mergers" due to the fact that their gas is heated and stripped by shock heating in the hot virial halo of the massive galaxy (e.g., Khochfar \& Silk 2009; Hopkins et al. 2010). These general results are also found by Naab et al. (2007, 2009).

With a mass of $\log M_{\text {vir }} / M_{\odot}>13$, galaxy G1 is well above the "critical mass" where the cooling time of the gas is much longer than the dynamical time, such that an accretion shock is established near the virial radius (e.g., Birnboim \& Dekel 2003; Dekel \& Birnboim 2006; Kereš et al. 2009; van de Voort $\&$ Schaye 2012). The rate at which the cooling time increases with decreasing redshift $\left(\tau_{\text {cool }} \propto 1 / \rho \propto(1+z)^{-3}\right)$ is more rapid than the increase in the dynamical time with decreasing redshift, $\left(\tau_{\text {dyn }} \propto 1 / \sqrt{\rho} \propto(1+z)^{-3 / 2}\right)$. Thus, it is generally found in simulations that, at high redshifts, cold streams can often penetrate the hot halos and accrete onto the galaxies, whereas at intermediate to low redshifts, the longer cooling time results in the heating and shocking of the cold filaments, which then accrete into the halo but not onto the galaxy (Kereš et al. 2005; Dekel \& Birnboim 2006; Faucher-Giguère et al. 2011; van de Voort et al. 2011). Galaxy G1 may have experienced the process in which it formed stars early during its initial accretion, perhaps even experiencing cold accretion from the filament that we are observing, and then at later times the accretion penetrated no further than into the halo, resulting in a high-mass, metal-rich, early-type galaxy with quenched star formation.

Given such a scenario, the H I complex could be interpreted as a filamentary structure with IGM chemical enrichment levels that is undergoing shock disruption near the viral radius of galaxy G1. The hot cloud (cloud 11) exhibits the signature of the shocked portion of the filament, and the multi-phase absorbers (clouds 8-10) exhibit the signature of a conductive interface. Galaxy G1 is probed by the quasar sight line impact parameter at $D / R_{\text {vir }} \simeq 0.1$. Simulations indicate that "cold" filaments (i.e., those that have not been heated above $\log T=5.5$ ) penetrate no deeper than $R \simeq 0.5 R_{\text {vir }}$ at intermediate redshifts (Kereš et al. 2009; van de Voort et al. 2011), suggesting that the sight line is intercepting the filament in the outer part of the virialized halo.

A schematic model of this interpretation is illustrated in Figure 9. The schematic is consistent with simulation results and is guided by inspection of Figure 7 (top panel, for a $z=2$, $M_{\mathrm{vir}}=10^{12} M_{\odot}$ galaxy) from van de Voort et al. (2011) and the "cold only" panel of Figure 6 for a $z=1, M_{\text {vir }}=10^{13} M_{\odot}$ galaxy from Kereš et al. (2009). 
Presumably, any components of the filament that have not yet been shocked will be shock heated and their cooling time will increase to be on the order of the Hubble time (as is deduced for cloud 11). Those components that may reside within the shock radius will likely not survive past $R \simeq 0.5 R_{\text {vir }}$ and be assimilated into the hot halo. This would imply that, ultimately, the filament will accrete into the halo, be heated to the halo temperature, and never accrete onto the galaxy itself. Galaxy G1 has likely not had new gas to fuel star formation for several gigayears and will likely not acquire new gas via accretion as it evolves. Apart from the possibility of dry minor mergers (Khochfar \& Silk 2009; Hopkins et al. 2010; Johansson et al. 2012) building the stellar mass of galaxy G1, it is likely that the galaxy has experienced secular stellar evolution since $z=4$.

If galaxy G3 is at $z \simeq 0.67$, one might ask if the filament is associated with this lower mass $\log M_{\mathrm{vir}} / M_{\odot} \simeq 11.9$ galaxy. With the data in hand, it is nearly impossible to claim any relationship between galaxy G3, the H i complex, and galaxy G1. However, a virial shock is not predicted for lower mass galaxies, which have cooling times shorter than their dynamical times. As such, it would be expected that a filament accreting onto galaxy G3 would not show the signature of shocked gas with conductive interfaces as seen in the $\mathrm{H}$ I complex. It could be speculated that galaxy G3 is embedded in the filament, perhaps contributing to some in situ metal enrichment, and might eventually be a dry minor merger (2\% mass ratio) with galaxy G1.

This scenario for the evolution of galaxy G1 is in stark contrast to the evolution of spiral galaxy G2, which is observed to have ongoing star formation at $z=0.661$, likely due to ongoing multi-phase accretion that is feeding the galaxy (Kacprzak et al. 2012). To the limit of $\log N\left(\mathrm{H}_{\mathrm{I}}\right) \simeq 12.4$, there is no indication in the COS spectrum of absorbing gas that bridges these two galaxies, which are separated by $\Delta v=1960 \mathrm{~km} \mathrm{~s}^{-1}$ (in the frame of the galaxies).

The scenario of a shock-heated filament for which the accretion onto galaxy G1 is quenched is very different to the favored scenarios for the lower redshift $\mathrm{H}$ I complexes toward H1821+643, PKS 2155-304, and HS 0624+6907 (Shull et al. 1998, 2003; Tripp et al. 2001; Aracil et al. 2006a). None of these complexes is in the vicinity of a massive elliptical galaxy, and as such it would be expected that they represent physical scenarios other than the one we propose for the Q1317+277 H I complex. That is, H I absorbing complexes, though filamentary in nature, likely trace various environments around galaxies and their relationships with the IGM.

\section{CONCLUSIONS}

We have studied the gas properties of and the luminous environment around a remarkable $\mathrm{H}$ I absorbing complex over the range $z=0.6686-0.6768$ having a velocity spread of $\Delta v=1600 \mathrm{~km} \mathrm{~s}^{-1}$ toward the quasar Q1317+277. To constrain the gas absorption properties, we have analyzed COS, STIS, and HIRES spectra of the quasar to examine the Lyman series, Mg II, $\mathrm{C}$ IV, and $\mathrm{O}$ VI absorption using VP fitting and photo+collisional ionization modeling. The galaxy properties in the quasar field have been measured using a WFPC2/F702W image, and multiband APO/SPIcam and KPNO/IRIM ground-based images. Stellar population modeling and halo abundance matching were employed to estimate the stellar ages, metallicities, mass, virial mass, radii, and temperatures of the galaxies.

Absorption properties. The H I complex is characterized by five contiguous absorbing regions, comprising 21 subcomponents, or clouds. Ionization models suggest that the kinematics are not due to the local Hubble flow, but are consistent with a $\sim 2$ Mpc by $\sim 200 \mathrm{kpc}$ structure with a total gas mass on the order of $10^{11} M_{\odot}$. We find a hot $\log T=5.7$ cloud, which we attribute to shock-heated gas. This cloud is bordered by multi-phase, cool, warm, and hot layers gas suggestive of a conductive interface in which metal line absorption ( $\mathrm{C} \mathrm{III}, \mathrm{CIV}$, and $\mathrm{O}$ VI) is detected. The low metallicity of the gas $\left(-2.5 \leqslant \log Z / Z_{\odot} \leqslant-1.7\right)$ is more consistent with enrichment levels of the high-redshift IGM (e.g., Cowie \& Songaila 1998; Simcoe et al. 2004), perhaps with low levels of in situ enrichment, than with the metallicities observed in the intracluster medium at $z=0.7$ (Balestra et al. 2007; Maughan et al. 2008).

Galaxy properties. We find that the galaxy G1 $(z=0.6719$ at impact parameter $D=58 \mathrm{kpc}$ ) is consistent with a massive $\left(\log M_{\mathrm{vir}} / M_{\odot} \simeq 13.7\right)$ elliptical galaxy that is metal rich ( $\left.Z \simeq Z_{\odot}\right)$, and formed at $z=4$ (6 Gyr stellar population). The magnitudes and colors of the other galaxies in the field, limits on the X-ray luminosity, and the low metallicity of the $\mathrm{H}$ I absorbing gas are consistent with galaxy G1 being a field galaxy with an evolutionary scenario in which the cold accretion has been shut down by shock heating. The redshift of galaxy G1 $(z=0.6719)$ is bracketed by the redshifts of the collisionally ionized gas $\left(z=0.6723, \Delta v=+76 \mathrm{~km} \mathrm{~s}^{-1}\right)$ and the multi-phase gas with detected metals consistent with a conductive interface $\left(z=0.6712-0.6717, \Delta v=-124\right.$ to $\left.-30 \mathrm{~km} \mathrm{~s}^{-1}\right)$ in the $\mathrm{H}_{\mathrm{I}}$ complex.

Interpretation. Based upon our data and analysis, we favor the scenario in which the H I complex is a filamentary structure accreting into the halo of galaxy G1 that is experiencing virial shock heating and dynamical disruption. Consistent with predictions of both theoretical treatments (e.g., Birnboim \& Dekel 2003; Dekel \& Birnboim 2006; Birnboim et al. 2007) and cosmological simulations (e.g., Kereš et al. 2005, 2009; Khochfar \& Silk 2009; Hopkins et al. 2010; Faucher-Giguère et al. 2011; van de Voort et al. 2011; van de Voort \& Schaye 2012; Johansson et al. 2012) of massive galaxies (i.e., $\log M_{\mathrm{vir}} / M_{\odot}>$ 12), our observations and analysis indicate that the gas accreting into the halo of galaxy G1 at times after its formation epoch have not accreted onto the galaxy itself and that the H I complex will also not accrete onto the galaxy. As such, the star formation of galaxy G1 has likely been quenched for gigayears.

Though it is difficult to definitively determine the nature of the $\mathrm{H}_{\mathrm{I}}$ absorbing complex, the scenario we favor is highly plausible and consistent with simulations and theory. In fact, the data appear to provide convincing evidence that theory and simulations correctly predict by $z<1$ that cold accretion via filaments in high-mass galaxies is shock heated as it accreted into the virialized hot halos and that this gas then grows the halos, but does not fuel further star formation. Continued growth of the stellar mass would then necessarily occur via dry mergers of minor galaxies.

The anonymous referee is gratefully acknowledged for helpful comments to clarify points, and for carefully reading this manuscript. We thank Daniel Ceverino for several stimulating and informative discussions during his visit to New Mexico State University and for helpful comments on an early draft of this paper, Kyle Stewart for several informative email exchanges with regard to halo abundance matching methods, and Avishai Dekel for helpful comments on cold streams penetrating the virialized shock-heated medium in massive halos. This research was primarily support through grant HST-GO-11667.01-A provided by NASA via the Space Telescope Science Institute, which is 
operated by the Association of Universities for Research in Astronomy (AURA) under NASA contract NAS 5-26555. C.W.C. thanks G.G.K., and Michael T. Murphy, and Swinburne Faculty Research Grants for providing funding for a visit to Swinburne University of Technology. Some observations are obtained with the Apache Point Observatory $3.5 \mathrm{~m}$ telescope, which is owned and operated by the Astrophysical Research Consortium (ARC). Additional data were obtained at Kitt Peak National Optical Astronomy Observatory, which is operated by AURA under cooperative agreement with the National Science Foundation. Some data presented herein were obtained at the W. M. Keck Observatory, which is operated as a scientific partnership among the California Institute of Technology, the University of California and NASA. The Observatory was made possible by the generous financial support of the W. M. Keck Foundation. The authors recognize and acknowledge the very significant cultural role and reverence that the summit of Mauna Kea has always had within the indigenous Hawaiian community. This research has made use of the NASA/IPAC Extragalactic Database (NED) which is operated by the Jet Propulsion Laboratory, California Institute of Technology, under contract with NASA. This research has also made use of the SIMBAD database, operated at Centre de Données, Strasbourg, France.

Facilities: HST (WFPC2, STIS, COS), Keck:I (HIRES, LRIS), ARC (3.5-m Telescope), Mayall (IRIM)

\section{REFERENCES}

Abraham, R. G., van den Bergh, S., Glazebrook, K., et al. 1996, ApJS, 107, 1 Aracil, B., Tripp, T. M., Bowen, D. V., et al. 2006a, MNRAS, 367, 139 Aracil, B., Tripp, T. M., Bowen, D. V., et al. 2006b, MNRAS, 372, 959 Asplund, M., Grevesse, N., Sauval, A. J., \& Scott, P. 2009, ARA\&A, 47, 481 Bahcall, J. N., Bergeron, J., Boksenberg, A., et al. 1993, ApJS, 87, 1 Bahcall, J. N., Bergeron, J., Boksenberg, A., et al. 1996, ApJ, 457, 19 Bahcall, J. N., \& Salpeter, E. E. 1965, ApJ, 142, 1677

Balestra, I., Tozzi, P., Ettori, S., et al. 2007, A\&A, 462, 429

Barlow, T. A., \& Sargent, W. L. W. 1997, AJ, 113, 136

Behroozi, P. S., Conroy, C., \& Wechsler, R. H. 2010, ApJ, 717, 379

Bell, E. F., McIntosh, D. H., Katz, N., \& Weinberg, M. D. 2003, ApJS, 149,289

Bertin, E., \& Arnouts, S. 1996, A\&AS, 117, 393

Binney, J. 1977, ApJ, 215, 483

Birnboim, Y., \& Dekel, A. 2003, MNRAS, 345, 349

Birnboim, Y., Dekel, A., \& Neistein, E. 2007, MNRAS, 380, 339

Brinchmann, J., \& Ellis, R. S. 2000, ApJ, 536, L77

Bruzual, G., \& Charlot, S. 2003, MNRAS, 344, 1000

Bryan, G. L., \& Norman, M. L. 1998, ApJ, 495, 80

Chabrier, G. 2003, PASP, 115, 763

Churchill, C. W. 1997, PhD dissertation, Univ. Califormia, Santa Cruz

Churchill, C. W., Kacprzak, G. G., Nielsen, N. M., Steidel, C. C., \& Murphy, M. T. 2012, ApJ, submitted

Churchill, C. W., Kacprzak, G. G., Steidel, C. C., \& Evans, J. L. 2007, ApJ, 661, 714 (Paper I)

Churchill, C. W., Mellon, R. R., Charlton, J. C., et al. 2000, ApJS, 130, 91

Churchill, C. W., Rigby, J. R., Charlton, J. C., \& Vogt, S. S. 1999b, ApJS, 120,51

Churchill, C. W., \& Vogt, S. S. 2001, AJ, 122, 679

Churchill, C. W., Vogt, S. S., \& Charlton, J. C. 2003, AJ, 125, 98

Conroy, C., \& Wechsler, R. H. 2009, ApJ, 696, 620

Cowie, L. L., \& Songaila, A. 1998, Nature, 394, 44

Cowie, L. L., Songaila, A., Hu, E. M., \& Cohen, J. G. 1996, AJ, 112, 839

Crain, R. A., McCarthy, I. G., Schaye, J., Frenk, C. S., \& Thuens, T. 2011, MNRAS, submitted (arXiv:1011.1906)

Davé, R., Hernquist, L., Katz, N., \& Weinberg, D. H. 1999, ApJ, 511, 521

Dekel, A., \& Birnboim, Y. 2006, MNRAS, 368, 2

Dekel, A., Birnboim, Y., Engel, G., et al. 2009, Nature, 457, 451

Ding, J., Charlton, J. C., \& Churchill, C. W. 2005, ApJ, 621, 615

Dinshaw, N., \& Impey, C. D. 1996, ApJ, 458, 73

Dixon, W. V., et al. 2011, Cosmic Origins Spectrograph Instrument Handbook, Version 4.0 (Baltimore, MD: STScI)
Dopita, M. A., \& Sutherland, R. S. 2003, Astrophysics of the Diffuse Universe (Berlin: Springer)

Draine, B. T. 2011, Physics of the Interstellar and Intergalactic Medium (Princeton, NJ: Princeton Univ. Press)

Ely, J., Aloisi, A., Bohlin, R., et al. 2011, STIS Instrument Handbook, Version 11.0 (Baltimore, MD: STScI)

Erb, D. K., Steidel, C. C., Shapley, A. E., et al. 2006, ApJ, 646, 107

Faber, S. M., Willmer, C. N. A., Wolf, C., et al. 2007, ApJ, 665, 265

Faucher-Giguère, C.-A., Kereš, D., \& Ma, C.-P. 2011, MNRAS, 417, 2982

Fontana, A., Pozzetti, L., Donnarumma, I., et al. 2004, A\&A, 424, 23

Gabor, J. M., \& Davé, R. 2012, MNRAS, in press (arXiv:1202.5315)

Ganguly, R., Eracleous, M., Charlton, J. C., \& Churchill, C. W. 1999, AJ, 117, 2594

Gibson, R. R., Jiang, L., Brandt, W. N., et al. 2009, ApJ, 692, 758

Haardt, F., \& Madau, P. 2012, ApJ, 746, 125

Hanson, R. 1986, SIAM J. Sci. Stat. Comput., 7, 826

Hopkins, P. F., Bundy, K., Hernquist, L., Wuyts, S., \& Cox, T. J. 2010, MNRAS, 401, 1099

Jakobsen, P., Perryman, M. A. C., di Serego Alighieri, S., Ulrich, M. H., \& Macchetto, F. 1986, ApJ, 303, L27

Jannuzi, B. T., Hartig, G. F., Kirhakos, S., et al. 1996, ApJ, 470, L11

Jarosik, N., Bennett, C. L., Dunkley, J., et al. 2011, ApJS, 192, 14

Johansson, P. H., Naab, T., \& Ostriker, J. P. 2012, ApJ, 754, 115

Kacprzak, G. G., Churchill, C. W., Evans, J. L., Murphy, M. T., \& Steidel, C. C 2011, MNRAS, 416, 3118

Kacprzak, G. G., Churchill, C. W., Steidel, C. C., et al. 2012, MNRAS, in press (arXiv:1208.4098)

Kannappan, S. J. 2004, ApJ, 611, L89

Kereš, D., Katz, N., Fardal, M., Davé, R., \& Weinberg, D. H. 2009, MNRAS, 395,160

Kereš, D., Katz, N., Weinberg, D. H., \& Davé, R. 2005, MNRAS, 363, 2

Khochfar, S., \& Silk, J. 2009, MNRAS, 397, 506

Kriss, G. A. 2011, COS Instrument Handbook, Version 01 (Baltimore, MD: STScI)

Lawton, B., Churchill, C. W., York, B. A., et al. 2008, AJ, 136, 994

Lequeux, J. 2005, The Interstellar Medium (Berlin: Springer)

Maughan, B. J., Jones, C., Forman, W., \& Van Speybroeck, L. 2008, ApJS, 174, 117

McGaugh, S. S. 2005, ApJ, 632, 859

Mickaelian, A. M., Hovhannisyan, L. R., Engels, D., Hagen, H.-J., \& Voges, W. 2006, A\&A, 449, 425

Moster, B. P., Somerville, R. S., Maulbetsch, C., et al. 2010, ApJ, 710 903

Mullis, C. R. 2001, PhD thesis, IfA, Univ. Hawaii

Naab, T., Johansson, P. H., \& Ostriker, J. P. 2009, ApJ, 699, L178

Naab, T., Johansson, P. H., Ostriker, J. P., \& Efstathiou, G. 2007, ApJ, 658, 710

Ocvirk, P., Pichon, C., \& Teyssier, R. 2008, MNRAS, 390, 1326

Osterbrock, D. E., \& Ferland, G. J. 2006, Astrophysics of Gaseous Nebulae and Active Galactic Nuclei (Mill Valley, CA: University Science Books)

Rauch, M. 1998, ARA\&A, 36, 267

Rees, M. J., \& Ostriker, J. P. 1977, MNRAS, 179, 541

Ribaudo, J., Lehner, N., Howk, J. C., et al. 2011, ApJ, 743, 207

Savage, B. D., \& Sembach, K. R. 1991, ApJ, 379, 245

Schaye, J. 2001, ApJ, 559, 507

Schlegel, D. J., Finkbeiner, D. P., \& Davis, M. 1998, ApJ, 500, 525

Schneider, D. P., Hartig, G. F., Jannuzi, B. T., et al. 1993, ApJS, 87, 45

Sembach, K. R., Wakker, B. P., Savage, B. D., et al. 2003, ApJS, 146, 165

Shaw, B., et al. 2009, COS Data Handbook, Version 1.0 (Baltimore, MD: STScI)

Shull, J. M., Penton, S. V., Stocke, J. T., et al. 1998, AJ, 116, 2094

Shull, J. M., Tumlinson, J., \& Giroux, M. L. 2003, ApJ, 594, L107

Silk, J. 1977, ApJ, 211, 638

Simard, L., Willmer, C. N. A., Vogt, N. P., et al. 2002, ApJS, 142, 1

Simcoe, R. A., Sargent, W. L. W., \& Rauch, M. 2004, ApJ, 606, 92

Steidel, C. C., Dickinson, M., \& Persson, S. E. 1994, ApJ, 437, L75

Steidel, C. C., \& Sargent, W. L. W. 1992, ApJS, 80, 1

Stewart, K. R. 2011, arXiv:1109.3207v1

Stewart, K. R., Bullock, J. S., Wechsler, R. H., \& Maller, A. H. 2009, ApJ, 702, 307

Strader, J., Romanowsky, A. J., Brodie, J. P., et al. 2011, ApJS, 197, 33

Strutskie, M. F., Cutri, R. M., Stiening, R., et al. 2006, AJ, 131, 1163

Sutherland, R. S., \& Dopita, M. A. 1993, ApJS, 88, 253

Swindle, R., Gal, R. R., La Barbera, F., \& de Carvalho, R. R. 2011, AJ, 142 118

Thom, C., Werk, J. K., Tumlinson, J., et al. 2011, ApJ, 736, 1

Tripp, T. M., Giroux, M. L., Stocke, J. T., Tumlinson, J., \& Oegerle, W. R. 2001, ApJ, 563, 724 
Trujillo-Gomez, S., Klypin, A., Primack, J., \& Romanowsky, A. J. 2011, ApJ, 742,16

van de Voort, F., \& Schaye, J. 2012, MNRAS, 423, 2991

van de Voort, F., Schaye, J., Booth, C. M., Haas, M. R., \& Dalla Vecchia, C. 2011, MNRAS, 414, 2458 van der Wel, A., Franx, M., van Dokkum, P. G., et al. 2005, ApJ, 631, 145

Verner, D. A., \& Iakovlev, D. G. 1990, Ap\&SS, 165, 27

Voges, W., Aschenbach, B., Boller, T., et al. 1999, A\&A, 349, 389

Weymann, R. J., Morris, S. L., Foltz, C. B., \& Hewett, P. C. 1991, ApJ, 373, 23

White, S. D. M., \& Rees, M. J. 1978, MNRAS, 183, 341 